\title{
Enhanced canonical Wnt signaling during early zebrafish development perturbs the interaction of cardiac mesoderm and pharyngeal endoderm and causes thyroid specification defects
}

Isabelle Vandernoot ${ }^{1, *}$, Benoît Haerlingen ${ }^{1, *}$, Achim Trubiroha ${ }^{1,2}$, Pierre Gillotay ${ }^{1}$, Véronique Janssens ${ }^{1}$, Robert Opitz ${ }^{1,3, \#}$ and Sabine Costagliola ${ }^{1, \#}$

${ }^{1}$ Institute of Interdisciplinary Research in Molecular Human Biology (IRIBHM), Université Libre de Bruxelles, Route de Lennik 808, 1070 Brussels, Belgium.

${ }^{2}$ German Federal Institute for Risk Assessment (BfR), Department Chemicals and Product Safety, Max-Dohrn-Strasse 8-10, 10589, Berlin, Germany.

3 Institute of Experimental Pediatric Endocrinology, Charité Universitätsmedizin Berlin, Augustenburger Platz 1, 13353, Berlin, Germany.

* These authors contributed equally to this work.

\# These authors contributed equally to this work.

\section{Author Contact Details:}

Isabelle Vandernoot Isabelle.Vandernoot@erasme.ulb.ac.be

Benoit Haerlingen benoit.haerlingen@gmail.com

Achim Trubiroha achim.trubiroha@bfr.bund.de

Pierre Gillotay $\quad$ Pierre.Gillotay@ulb.ac.be

Véronique Janssens verojans@ulb.ac.be

Robert Opitz robert.opitz@charite.de

Sabine Costagliola scostag@ulb.ac.be

Correspondence and requests for materials should be addressed to:

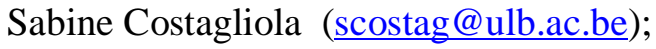

IRIBHM, Université Libre de Bruxelles, Campus Erasme, Bat. C., 808 route de Lennik, B1070 Brussels, Belgium.

Robert Opitz (robert.opitz@ charite.de);

Institute of Experimental Pediatric Endocrinology, Charité Universitätsmedizin Berlin, Augustenburger Platz 1, 13353, Berlin, Germany. 


\section{$1 \quad \underline{\text { Abstract }}$}

2 Background: Congenital hypothyroidism $(\mathrm{CH})$ due to thyroid dysgenesis is a frequent

3 congenital endocrine disorder for which the molecular mechanisms remain unresolved in the

4 far majority of cases. This situation reflects in part our still limited knowledge about the

5 mechanisms involved in the early steps of thyroid specification from the endoderm, in

6 particular the extrinsic signaling cues that regulate foregut endoderm patterning. In this study,

7 we used small molecules and genetic zebrafish models to characterize the role of various

8 signaling pathways in thyroid specification.

9 Methods: We treated zebrafish embryos during different developmental periods with small

10 molecule compounds known to modulate the activity of Wnt signaling pathway and observed

11 effects in thyroid, endoderm and cardiovascular development using whole mount in situ

12 hybridization and transgenic fluorescent reporter models. We used an antisense morpholino

13 to create a zebrafish acardiac model. For thyroid rescue experiments, BMP pathway induction

14 in zebrafish embryos was obtained by using heatshock inducible transgenic lines.

15 Results: Interestingly, combined analyses of thyroid and cardiovascular development revealed that overactivation of Wnt signaling during early development leads to impaired thyroid

17 specification concurrent with severe defects in the cardiac specification. When using a model 18 of morpholino-induced blockage of cardiomyocyte differentiation, a similar correlation was 19 observed, suggesting that defective signaling between cardiac mesoderm and endodermal 20 thyroid precursors contributes to thyroid specification impairment. Rescue experiments 21 through transient overactivation of BMP signaling could partially restore thyroid specification 22 in models with defective cardiac development.

23 Conclusion: Collectively, our results indicate that BMP signaling is critically required for 24 thyroid cell specification and identify cardiac mesoderm as a likely source of BMP signals. 


\section{Introduction}

The thyroid is an endoderm-derived gland developing from the most anterior part of the gut tube. The thyroid organogenesis starts with the specification of its anlage, a group of thyroid precursor cells that are characterized by co-expression of a unique combination of transcription factors comprising NKX2-1, PAX8, FOXE1, and HHEX (1).

The median anlage develops into a diverticulum that evaginates and loses contact with the ventral foregut endoderm to relocate deeper into the subpharyngeal mesenchyme. Terminal differentiation, which leads to functional follicle formation, is initiated during the migration of the thyroid primordium (2).

Congenital hypothyroidism $(\mathrm{CH})$ is the most frequent congenital endocrine disorder, affecting approximately one of 2500 in human newborns (3). $\mathrm{CH}$ is characterized by reduced serum thyroid hormone levels at birth. It is caused in $85 \%$ of the cases by thyroid dysgenesis (TD due to ectopy, athyreosis, or hypoplasia, resulting from an aberration of the thyroid gland embryological development. The molecular mechanisms leading to TD are mostly unknown, with mutations in the known thyroid transcription factors explain only $5 \%$ of TD cases (1). It probably reflects our limited knowledge about intrinsic factors and external signals coordinating thyroid organogenesis and suggests that unknown genetic and/or epigenetic factors might be crucial for thyroid development (4).

Zebrafish is a valuable model system that has already helped us to improve further our understanding of morphogenetic processes and gene networks involved in thyroid organogenesis (5). Over the past two decades, zebrafish has gained much attention as a genetically tractable vertebrate model to study organogenesis (6). Embryos' optical clarity allows direct visualization of developmental processes and their pathological deviations. Small size, high fecundity, external fertilization, rapid development, and short generation time are important attributes underscoring the utility of zebrafish study of early developmental 
51 processes. The value of zebrafish for studies on thyroid development is supported by the fact

52 that several aspects of thyroid morphogenesis are well conserved between zebrafish and

53 mammals (7), (8). Early morphogenetic events, such as thyroid specification, budding, and

54 relocalization into the subpharyngeal mesenchyme, show many similarities in fish and mouse

55 (reviewed in ref. (9)). Moreover, the developing thyroid expresses a similar, but not identical,

56 set of transcription factors in mouse (NKX2-1, PAX8, HHEX, FOXE1 (1)) and zebrafish

57 (nkx2.4b (10), pax2a, pax8, foxe1, hhex (7), (10)) embryos. Invalidation of the known thyroid

58 transcription factors in mice leads to athyreosis (NKX2-1, PAX8, FOXE1, HHEX) or thyroid

59 ectopy (FOXE1) (11). Zebrafish with loss-of-function of $n k x 2.4 b$, pax2a, and hhex similarly

60 display athyreosis (7), (9), (12). However, differences exist in the timing of specific

61 morphogenetic events and the anatomy of the mature thyroid tissue. Indeed, although thyroid

62 follicles are encapsulated in a compact organ in mouse, thyroid follicles are loosely scattered

63 along the pharyngeal midline in zebrafish.

64 In this study, we mainly focused on molecular mechanisms that govern the first steps

65 of thyroid specification. At present, the signals that trigger the specification of pharyngeal

66 endodermal cells into thyroid precursors remains unknown. Recent studies demonstrate that

67 tissue-tissue interactions, in particular with the cardiac mesoderm and pharyngeal blood

68 vessels, are essential for correct thyroid development (13), (5). Defective pharyngeal vessel

69 development has been shown to cause thyroid anomalies in mice and zebrafish (14), (15).

70 There are also pieces of evidence coming from human disease studies since an increased

71 prevalence of congenital cardiovascular anomalies is observed in patients with thyroid

72 dysgenesis compared to the healthy population (16). However, the mechanisms underlying

73 tissue-tissue interactions during thyroid development (particularly between the developing

74 thyroid and the cardiac mesoderm and pharyngeal vessels) and the signals involved in this

75 crosstalk are poorly understood. While a crucial role of FGF expression in the mesoderm 
surrounding the developing thyroid has been demonstrated in mouse and zebrafish (17), (18), the potential functions of other major signaling pathways in thyroid organogenesis is still to be clarified (2), (19). A significant advantage of the zebrafish model in cardiovascular development studies is that zebrafish embryos and larvae can survive for several days without a functional heart or in the absence of blood circulation (20). This facilitates the characterization of developmental effects over an extended developmental period, compared to most mouse models with cardiovascular defects.

To improve our understanding of the role of extrinsic signaling cues in thyroid development, we recently exploited the amenability of zebrafish embryos for small molecule screenings to identify candidate signaling pathways (12), (21). From a literature review, we first identified a collection of small molecules known to interfere with common signaling pathways in zebrafish. The pharmacological screening readily identified modulators of Wnt, BMP, FGF, and TGF- $\beta$ signaling to cause distinct effect patterns of disturbed thyroid organogenesis (e.g., agenesis, hypoplasia, ectopy) (21). In this paper, we focused on the phenotypic description, using thyroid and cardiovascular markers, of early thyroid development after Wnt modulation and propose a mechanistic explanation.

Wnt signaling pathway is an evolutionarily conserved signal transduction pathway that regulates crucial aspects of cell fate determination, cell migration, cell polarity and cell differentiation during embryonic development (22). Wnt proteins are secreted glycoproteins that bind to the N-terminal extra-cellular cysteine-rich domain of the Frizzled (Fz) receptor family, interacting with the LRP5/6 co-receptors. To date, several intracellular signaling branches/cascades downstream of the Fz receptors have been identified including a canonical ( $\beta$-catenin dependent) pathway and a non-canonical ( $\beta$-catenin-independent) pathway. Some Wnt ligands act preferentially on one pathway or the other, but the activated pathway mostly depends on the cellular context. Without Wnt canonical signaling activation, cytoplasmic $\beta$ - 
101 catenin is degraded by a $\beta$-catenin destruction complex, which includes Axin, adenomatosis

102 polyposis coli (APC), protein phosphatase 2A, glycogen synthase kinase 3 (GSK3) and

103 casein kinase $1 \alpha$ (CK1). Binding of a Wnt ligand to its receptor leads to a series of events

104 that disrupt the $\beta$-catenin destruction complex, which is required for the $\beta$-catenin

105 translocation into the nucleus and activation of target genes (23), (24).

106 The functions of $\mathrm{Wnt} / \beta$-catenin signaling in embryogenesis have been extensively

107 studied in different animal models. It plays a crucial biphasic role in heart development, as

108 demonstrated in in vitro cells and zebrafish: in pregastrula stages, canonical Wnt promotes the

109 specification of the precardiac mesoderm into cardiomyocyte progenitors, but it inhibits the

110 cardiac differentiation of these cells during gastrula stages (25), (26). It has already been

111 demonstrated that this pathway also plays a very important role in the specification and

112 development of endoderm-derived organs, like liver and pancreas. An anterior-posterior

113 gradient of Wnt activity in Xenopus plays a crucial role in endodermal patterning, the anterior

114 endoderm giving rise among others to liver, lung, and thyroid, the posterior endoderm leading

115 to intestinal fate (27). At later stages in zebrafish, Poulain et al. have shown that Wnt pathway

116 is necessary for liver progenitors proliferation (28). However, very little data exist on the role

117 of Wnt in thyroid development and/or maintenance.

118 Therefore, this study aims to examine the effects of the canonical Wnt signaling

119 pathway on thyroid development in zebrafish, in regard to the adjacent cardiogenesis. We

120 used different chemicals and genetic models to modulate canonical Wnt signaling during

121 gastrula and early somitogenesis stages and observed effects of such modulation on thyroid

122 organogenesis. We succeeded to partially rescue thyroid defects obtained after Wnt activation

123 when combining this chemical treatment with induction of BMP pathway using heatshock

124 inducible transgenic lines. 


\section{Material and methods}

127 Zebrafish husbandry and embryo culture

128 Zebrafish (Danio rerio) embryos were obtained from natural spawning of adult fish, raised at $12928.5^{\circ} \mathrm{C}$ according to Westerfield (29) and staged in hours postfertilization (hpf) as described 130 by Kimmel et al.. (30) In addition to wild-type (31) and casper mutant lines (32), different

131 transgenic lines were used in this study: $\operatorname{Tg}$ (tg:mCherry) (5), $\operatorname{Tg}(\mathrm{kdrl}: E G F P)$ (33),

$132 \operatorname{Tg}(m y l 7: E G F P) \quad(34), \quad T g(7 x T C F-X l a . S i a m: G F P) i a 4 \quad$ (35), $\quad T g(\operatorname{sox} 17: E G F P) \quad(36)$,

$133 T g(h s p 70 l: b m p 2 b)$ (37) and Tg(hsp70l:wnt8a-EGFP) (38). Embryos were dechorionated at 24

134 hpf using $0.6 \mathrm{mg} / \mathrm{mL}$ pronase (Sigma), anesthetized in $0.016 \%$ tricaïne (Sigma), fixed in $4 \%$

135 phosphate-buffered paraformaldehyde (PFA; Sigma) overnight at $4^{\circ} \mathrm{C}$, washed in PBS

136 containing $0.1 \%$ Tween 20 (PBST), gradually transferred to $100 \%$ methanol, and stored at

$137-20^{\circ} \mathrm{C}$ until used for in situ hybridization or immunofluorescence analyses. If indicated,

138 pigmentation of embryos was inhibited by adding 0.003\% 1-phenyl-2-thiourea (PTU; Sigma)

139 (39) to the embryo medium at $24 \mathrm{hpf}$. All zebrafish work at the Institute of Interdisciplinary

140 Research in Molecular Human Biology followed protocols approved by the Institutional

141 Animal Care and Use Committee.

142

$143 \quad$ Small molecule treatment

144 We used timed embryo treatment with BIO (Sigma, B1686), 1-Azakenpaullone

145 [AZA] (Sigma, A3734), and IWR-1 (Sigma, I0161) to modulate the canonical Wnt signaling 146 pathway during distinct developmental periods. BIO and AZA act as canonical Wnt activators

147 due to GSK3 $\beta$ inhibition (40). In contrast, IWR-1 stabilizes the destruction complex, thereby 148 acting as an inhibitor of Wnt signaling (41). Stock solutions of BIO (5 mM), AZA (5 mM), 149 and IWR-1 (10 $\mathrm{mM})$ were prepared in dimethyl sulfoxide (DMSO). Test solutions were 
150 prepared by diluting the stock solutions in embryo medium. A control treatment containing

$1510,1 \%$ DMSO was used in all experiments involving a drug treatment.

152

Heat-shock treatments

154 Timed global overactivation of Wnt and BMP signaling was induced by heat-shock treatment

155 of the progeny from matings of WT fish with heterozygous $T g$ (hsp70l:wnt8a-GFP) (38) or

$156 \operatorname{Tg}(h s p 70 l: b m p 2 b)(37)$ fish, respectively. Embryos obtained from these matings were raised

157 under standard conditions until heat $\square$ shock to induce global overexpression of EGFP-tagged

158 Wnt8a or Bmp2b. For the heat-shock treatments, embryos were transferred to dishes

159 containing prewarmed embryo medium at $40^{\circ} \mathrm{C}$ and incubated in an incubator for $30 \mathrm{~min}$ at

$16040^{\circ} \mathrm{C}$. After heat-shock treatment, embryos were transferred to Petri dishes containing fresh

161 medium and allowed to develop further at $28.5^{\circ} \mathrm{C}$ under standard conditions. Carriers of the

162 hsp70l:wnt8a-EGFP transgene were identified 3 hours after heat-shock by means of their

163 EGFP expression. Embryos carrying the $h s p 70 l: b m p 2 b$ transgene were identified by their

164 dorsalized phenotype if heat-shocked at early somitogenesis or by PCR genotyping if

165 embryos were heat-shocked at later stages (15 or $20 \mathrm{hpf}$ ). For the latter, PCR genotyping was

166 performed after completion of WISH experiments as previously described (42) using the

167 following primers: Forward 5'-CATGTGGACTGCCTATGTTCATC-3' (primer located in

168 hsp70l promoter sequence); Reverse 5'-GAGAGCGCGGACCACGGCGAC-3' (primer

169 located in bmp $2 b$ coding sequence).

170

Whole-mount in situ hybridization (WISH)

172 DNA templates for synthesis of $n k x 2.4 b, t g, n k x 2.5$, egfp, mef2cb, gata4, gata5, hhex,

173 foxa2, foxa3, pdx1, proxl, and bmp4 riboprobes were generated by PCR (see Supplemental

174 Table 1 for primer sequences). Plasmids for $a m h c, v m h c, m y l 7$, scl, and hand2 riboprobes 
175 have been used as described (43), (44), (45). Single-color WISH using digoxigenin (DIG)-

176 labeled riboprobes and anti-DIG antibody conjugated to alkaline phosphatase was performed

177 essentially as described in Thisse and Thisse (46) and Opitz et al (5). Riboprobe hybridization

178 was performed at $65^{\circ} \mathrm{C}$ overnight, and probes were detected using an anti-DIG antibody

179 (1:6000; Roche). Staining reactions were performed with the alkaline phosphatase substrates

180 BM Purple (Roche) or NBT/BCIP (Roche). For dual-color WISH, riboprobes labeled with

181 DIG, dinitrophenol (DNP), or fluorescein (FLU) were used, and sequential alkaline

182 phosphatase staining was performed with BM Purple and Fast Red (Sigma) as described

183 (21).We first detect the DIG or DNP riboprobes with anti-DIG or anti-DNP antibody using

184 BM Purple (for most genes) or NBT/BCIP (for $t g$ ) staining solution and used an anti-FLU

185 antibody in combination with FastRed for detection of the FLU probe in a second step.

186 Removal of the antibodies after the first staining step was performed by 2 x 5 min incubation

187 in $100 \mathrm{mM}$ glycine- $\mathrm{HCl}(\mathrm{pH} 2.2)$.

188 Fluorescent WISH (FISH) using a DIG-labeled riboprobe for $n k x 2.4 b$ was performed as

189 described (5). Antibodies used in WISH and FISH experiments are listed in Supplemental

190 Table 2. Stained embryos were washed in PBST, postfixed in 4\% PFA and embedded in 90\%

191 glycerol for whole-mount imaging or in $7 \%$ low melting point agarose (Lonza) for vibratome

192 sectioning. Whole-mount images of WISH and FISH were acquired using a MZ16F Leica

193 stereomicroscope equipped with a DFC420C camera or a Leica microscope DMI6000B

194 equipped with a DFC365FX camera, respectively. Vibratome tissue sections at 50-60 $\mu \mathrm{m}$

195 thickness were cut on a Leica VT1000S vibratome and mounted in Glycergel (Dako).

196 Confocal images of vibratome sections were acquired using an LSM510 confocal microscope

197 (Zeiss).

198

199 Whole-mount immunofluorescence (WIF) 
WIF was performed essentially as described (47). Briefly, after rehydration into

201 PBST, embryos were immersed in blocking buffer (PBST containing 1.0\% DMSO, 1\%

202 bovine serum albumin [BSA], 5\% horse serum, and $0.8 \%$ Triton X-100) for $2 \mathrm{~h}$. Embryos

203 were then incubated overnight in blocking buffer containing primary antibodies at $4^{\circ} \mathrm{C}$. After

204 several washing steps in PBST containing 1\% BSA, embryos were incubated with secondary

205 antibodies overnight at $4^{\circ} \mathrm{C}$. Specifications and sources of primary and secondary antibodies

206 used to detect EFGP green fluorescent protein (48) and pSMAD1/5/8 protein in zebrafish

207 embryos are provided in Supplemental Table 2. Stained embryos were washed in PBST and

208 embedded in 90\% glycerol for fluorescence microscopy. Images were acquired with a Leica

209 DFC365FX camera mounted on a Leica DMI6000B. Combined FISH and WIF staining was

210 performed as described (5). Confocal images were acquired using a LSM510 confocal

211 microscope (Zeiss).

212

$213 \quad$ RNA extraction and RT-qPCR

214 For total RNA preparation, pools of 10-20 embryos per sample were lysed in RNeasy

215 Lysis buffer (Qiagen) containing 1\% 2-mercaptoethanol (Sigma). Total RNA was isolated

216 using RNeasy RNA preparation microkit (Qiagen) according to the manufacturer's

217 instructions, including on-column treatment with DNase I (Qiagen). Reverse transcription

218 was done using Superscript II kit (Invitrogen). Reverse transcription quantitative PCR (RT-

219 qPCR) was performed in duplicate using Kapa SYBR Fast (KapaBiosystems) mix and a CFX

220 Connect Real-Time PCR System (Biorad).

221 Relative values for target transcript abundance in individual samples were determined by the

222 comparative $\mathrm{C}_{\mathrm{T}}$ method $\left(\Delta \Delta \mathrm{C}_{\mathrm{T}}\right)$ according to Pfaffl et al. (2001) (49), and data are presented

223 as relative expression values normalized to the reference gene rpl13 (NM_212784.1) whose

224 expression remained constant upon treatment. Primers used were as follows: rpll3, forward 
TCTGGAGGACTGTAAGAGGTATGC, reverse AGACGCACAATCTTGAGAGCAG (50); egfp, forward AGAACGGCATCAAGGTGAAC, reverse TGCTCAGGTAGTGGTTGTCG.

227 Gene expression profile was confirmed in triplicate (3 different batches of embryos). Data were expressed as mean \pm SD. Pairwise comparisons were performed using the Student $t$-test.

\section{Fluorescence-activated cell sorting}

Cell suspensions were prepared from $\operatorname{Tg}(\operatorname{sox} 17: E G F P)$ transgenic embryos at 30 hpf. Embryos were digested in HBSS (Gibco) containing 0.25\% trypsin (Gibco) and $2 \mathrm{mM}$

233 EDTA (Invitrogen). Single-cell solutions were obtained by constant pipetting. Reaction was 234 stopped by adding $\mathrm{CaCl} 2$ (final concentration: $1 \mathrm{mM}$ ) and $\mathrm{FBS}$ (final concentration: 10\%).

235 Cells were pelleted by centrifugation and washed with PBS containing $0.4 \%$ BSA and $2 \mathrm{mM}$ 236 EDTA. Finally, resuspended cells were passed through a $40 \mu \mathrm{M}$ nylon mesh (Falcon) into a 237 FACS tube (Falcon) and the GFP+ fractions were analyzed by a fluorescent-activated cell 238 sorter (FACS Aria: FACSDiva Software (BD)). About 50 embryos were sampled per 239 condition, and each experiment was performed in triplicate.

242 For inhibition of the mef $2 c a$ and $m e f 2 c b$ transcripts, zebrafish embryos were injected 243 with morpholino (MO) antisense oligonucleotides that have previously been validated for

244 their knockdown specificity and efficacy (51), (52). 3nL (2ng/nL) of a translation-blocking $245 m e f 2 d / c-\mathrm{MO}$ (tb-MO; 5'-ATGGGGAGGAAAAAGATCCAGATTC-3') was injected as

246 previously described (51). Working solutions of MOs were prepared in $0.12 \mathrm{M} \mathrm{KCl}$

247 containing phenol red, and $3 \mathrm{~nL}$ were microinjected into the yolk of one- to two-cell stage 248 embryos. Non-injected embryos served as control embryos. 


\section{Statistics}

For the statistical analyses of thyroid and cardiac phenotypes in the rescue experiments, Fisher tests were conducted using the software package GraphPad Prism 4.0 (GraphPad, San Diego, CA). Differences were considered significant at $\mathrm{p}<0,05$.

\section{$\underline{\text { Results }}$}

\section{Drug-induced manipulation of canonical Wnt signaling disrupts early thyroid development}

We recently performed a small molecule screen to identify signaling pathways involved in early zebrafish thyroid development (21). In these experiments, pharmacological manipulations of canonical Wnt signaling during gastrula stage resulted in abnormal thyroid marker expression, and most notably, in a severe reduction of $n k x 2.4 b$ expression at thyroid anlage stages ( $28 \mathrm{hpf}$ ) following overactivation of Wnt signaling.

To characterize in more detail the thyroidal effects resulting from enhanced Wnt signaling, we performed additional treatment experiments with $\mathrm{BIO}$ and AZA, two drugs known to activate $\mathrm{Wnt} / \beta$-catenin signaling (40). Consistent with our previous results, treatment of zebrafish embryos with BIO or AZA during the gastrulation period (6 to $10 \mathrm{hpf}$ ) caused concentration-dependent decreases of $n k x 2.4 b$ expression in 28 hpf embryos (Figure 1). Maximal effects on thyroidal $n k x 2.4 b$ expression (i.e. complete absence of a detectable WISH staining) were evident at $5 \mu \mathrm{M}$ BIO and $5 \mu \mathrm{M}$ AZA. In addition to the marked effects on the thyroidal $n k x 2.4 b$ expression domain, $\mathrm{BIO}$ and AZA treatments also caused concentration-dependent decreases in the size of the $n k x 2.4 b$ expression domain in the forebrain. The drug-induced effects on forebrain $n k x 2.4 b$ expression correlated closely with other visible signs of global posteriorization, including loss of anterior neural tissue and reduced size or absence of the eyes (53), (54). Although we did not quantify the relative size reductions of thyroidal and forebrain $n k x 2.4 b$ expression domains in 28 hpf embryos 
275 following different BIO and AZA treatments, we noted a similar sensitivity to drug treatment

276 for both expression domains when embryos were treated between $6-10 \mathrm{hpf}$.

277 Concentration-dependent effects on thyroid development were also evident when BIO-

278 and AZA-treated embryos were analyzed for thyroid marker expression at 55 hpf (Figure 2).

279 WISH analysis of $n k x 2.4 b$ expression revealed concurrent reductions in the size of thyroidal

280 and forebrain expression domains in treated embryos. Moreover, BIO and AZA treatment

281 strongly reduced the expression of the functional thyroid differentiation marker, thyroglobulin

282 ( $\operatorname{tg}$ ), in $55 \mathrm{hpf}$ embryos. However, we also noted that the majority of 55 hpf embryos treated

283 with high drug concentrations during gastrula stages displayed at least some residual staining

284 for $n k x 2.4 b$ and $t g$ despite undetectable thyroid marker expression at the thyroid anlage stage

285 (28 hpf). This was observed in all experiments involving drug treatment from 6 to $10 \mathrm{hpf}$.

286 Conversely, treatment of gastrulating embryos with $10 \mu \mathrm{M}$ IWR-1, a small molecule

287 inhibitor of canonical Wnt signaling, resulted in additional ectopic domains of thyroidal

$288 n k x 2.4 b$ and $t g$ expression (Supplementary figure 1). In $28 \mathrm{hpf}$ embryos, the additional

289 ectopic $n k x 2.4 b$ expression was detected posterior to the orthotopic thyroidal $n k x 2.4 b$

290 expression domain, whereas in 55 hpf embryos, supernumerary clusters of $t g$ expression were

291 detectable at irregular posterior positions. Taken together, these data indicate that the

292 canonical Wnt pathway negatively regulates thyroid specification during gastrulation stage in

293 zebrafish as drug-mediated activation or inhibition of Wnt activity during gastrulation impairs

294 or amplifies thyroid specification, respectively.

295

296 Small molecule compounds rapidly act on Wnt signaling

297 We next took advantage of an available Wnt signaling biosensor line, $\operatorname{Tg}(7 x T C F$ -

298 Xla.Siam:GFP) ${ }^{i a 4}$, to verify that the small molecule compounds BIO, AZA and IWR-1

299 effectively alter canonical Wnt signaling activities within a short space of time under the 
300

301

302

303

304

305

306

307

308

309

310

311

312

314

316

318

320

321

experimental conditions that resulted in irregular thyroid development. For this purpose, we treated Tg(7xTCF-Xla.Siam:GFP $)^{i a 4}$ embryos with BIO $(5 \mu \mathrm{M})$, AZA $(5 \mu \mathrm{M})$ or IWR-1 $(10$ $\mu \mathrm{M})$ from 6 to $10 \mathrm{hpf}$ and monitored the expression of GFP mRNA during the course of drug treatment by WISH and RT-qPCR (Supplementary figure 2).

WISH analyses of GFP mRNA expression in BIO- and AZA-treated embryos showed a rapid and robust up-regulation of GFP mRNA expression when compared to DMSO-treated embryos (0.1\% DMSO) indicating effective overactivation of canonical Wnt signaling in drugged embryos within 2 hours after the beginning of drug treatment. Consistently, IWR-1 treatment resulted in reduced GFP mRNA expression, evident within 2-3 hours after the beginning of drug treatment. Although several embryos treated with these small molecule compounds experienced slight developmental delay, the drug-induced alterations in reporter expression were robustly detectable in comparison with vehicle control embryos. In further experiments, we also corroborated the effects of BIO treatment on GFP mRNA expression by RT-qPCR. As shown in Supplementary figure 2, treatment of $T g(7 x T C F-X l a . S i a m: G F P)^{i a 4}$ embryos with $5 \mu \mathrm{M}$ BIO resulted in 2.5- to 3-fold increases of whole embryo expression levels of GFP mRNA within 2 hours after initiation of BIO treatment. Together, these data demonstrate that, under our experimental conditions, the drug treatments faithfully altered canonical Wnt signaling.

is due to transient overactivation of $\mathrm{Wnt} / \beta$-catenin signaling during gastrulation, we manipulated $\mathrm{Wnt} / \beta$-catenin signaling by heat-shock-induced overexpression of wnt $8 a$ in Tg(hsp70l:wnt8a-GFP) embryos (38). Wnt8a specifically activates Wnt/ $\beta$-catenin signaling in zebrafish embryos and a 30 min heat-shock of transgenic $T g(h s p 70 l$ :wnt $8 a-G F P)$ embryos at 6 hpf resulted in a marked neural posteriorization phenotype and disturbed early thyroid 
325 development (Figure 3). Non-transgenic embryos showed no discernible phenotypes in

326 response to the heat-shock treatment. The thyroid phenotype caused by transient wnt $8 a$

327 overexpression in the genetic model closely resembled the phenotypes observed following

328 treatment with 4-5 $\mu$ M BIO or AZA. Specifically, our WISH analyses failed to detect $n k x 2.4 b$

329 expression in the thyroid anlage region of heat-shocked embryos carrying the wnt $8 a-G F P$

330 transgene at $28 \mathrm{hpf}$ and a detectable, though strongly diminished, $t g$ expression in $55 \mathrm{hpf}$

331 transgenic embryos (Figure 3). Taken together, these experiments verified that the selected

332 drug concentrations cause overactivation of $\mathrm{Wnt} / \beta$-catenin signaling and that enhanced

$333 \mathrm{Wnt} / \beta$-catenin signaling during gastrula stages leads to defects in thyroid development.

334

Effects of early Wht overactivation on endoderm development

336 Our initial experiments with BIO and AZA showed that treatment with different drug

337 concentrations during gastrulation causes thyroid abnormalities and a strong posteriorization

338 of neural ectoderm. Considering that the latter phenotype is a well-characterized effect of

339 enhanced Wnt signaling during early development (55), we next examined whether

340 endodermal development is similarly affected by a global posteriorization activity due to

341 enhanced Wnt signaling and might thus explain the loss of thyroid marker expression in the

342 anterior endoderm.

343 To address this question, we first treated embryos of the transgenic $\operatorname{Tg}(\operatorname{sox} 17: E G F P)$

344 line with BIO $(5 \mu \mathrm{M})$ or AZA $(5 \mu \mathrm{M})$ between $6-10 \mathrm{hpf}$ and analyzed the gross

345 morphology of their foregut endoderm at different embryonic stages, in comparison to that of

346 DMSO-treated embryos . While a neural posteriorization phenotype (loss of anterior neural

347 tissue) was readily detectable in BIO- and AZA-treated $T g(\operatorname{sox} 17: E G F P)$ embryos, the gross

348 morphology of the anterior endoderm appeared unaffected in these embryos

349 (Supplementary figure 3). In addition, we used FACS to compare the number of GFP+ cells 
in $30 \mathrm{hpf} T g(\operatorname{sox} 17: E G F P)$ embryos following treatment with DMSO $(0.1 \%)$ and BIO

$351(5 \mu \mathrm{M})$ but did not detect a decrease in the number of GFP+ cells in BIO-treated embryos

352 (Supplementary figure 3). Thus, in contrast to the overt effects on anterior neural tissue

353 development, no visible dysgenesis of the anterior endoderm was detected in

$354 \operatorname{Tg}(\operatorname{sox} 17: E G F P)$ following early Wnt overactivation.

355 To further study possible global endodermal patterning defects in BIO and AZA-

356 treated embryos, we analyzed the expression of a panel of informative endodermal marker

357 genes by WISH (Figure 4). We first confirmed that thyroidal expression of $n k x 2.4 b$ and $h h e x$

358 is affected similarly by BIO and AZA treatment (see Figure 4A-D). In addition to the

359 thyroid, hhex is expressed in the developing liver and pancreas. Interestingly, hhex expression

360 was selectively repressed in the thyroid anlage of BIO-treated embryos and was maintained in

361 the liver/pancreas region (Figure 4D). Notably, the shape and position of the hepatic and

362 pancreatic hhex expression domains were altered in BIO-treated embryos. Therefore, we

363 analyzed the expression of foxa2, a master regulator of foregut endoderm patterning. Notably,

364 our WISH analyses did not reveal gross changes in the foxa2 anterior-posterior expression

365 pattern following BIO treatment (Figure 4E,F). However, when analyzing foxa3 expression,

366 a key regulator of posterior endoderm development, we noted that its expression domain

367 appeared extended more anteriorly in many embryos after BIO treatment (Figure 4G,H).

368 Analyses of $p d x 1$ and proxla expression confirmed that hepatic and pancreatic markers are

369 unaffected in BIO-treated embryos and that diminished expression in response to canonical

370 Wnt overactivation is limited to thyroidal marker genes (Figure 4I-L).

371 Collectively, our analyses indicate that the loss of thyroid marker expression caused

372 by overactivation of canonical Wnt signaling during gastrulation is not due to a general defect

373 in anterior foregut formation. However, as indicated by the irregular anterior foxa3 expression

374 in BIO-treated embryos and the posterior extension of thyroid marker expression in IWR-1- 
375 treated embryos, we cannot rule out that a Wnt-induced foregut endoderm posteriorisation

376 might contribute to the thyroid defects seen in the most anterior part of the foregut.

377

Cardiac phenotype is correlated with thyroid phenotype after gastrula Wnt activation

379 A recurrent theme in the development of endoderm-derived organs is the critical role

380 of tissue-tissue interactions, particularly between the endoderm and adjacent mesodermal

381 tissues (56), (28). Observations made during our initial small molecule screening experiments

382 (21) indicated that overactive Wnt signaling might induce the concurrent cardiac and

383 thyroidal maldevelopment. To examine this possible relationship in more detail, we first

384 confirmed that drug-induced overactivation of Wnt signaling during gastrula stages results in

385 a severe inhibition of heart formation (Supplementary figure 4) as previously reported for

386 genetic models with overactivation of canonical Wnt signaling (26), (25). When treated

387 between 6 to $10 \mathrm{hpf}$, effects of Wnt-activating drugs on heart development appeared

388 concentration-dependent, and we noticed that development of ventricular cardiomyocytes was

389 more severely affected, compared to that of atrial cardiomyocytes (Supplementary figure

390 4). Notably, the concentrations at which BIO and AZA caused severe cardiac

391 maldevelopment (Figure 5) overlapped the range at which repression of thyroid markers was

392 detected.

394 To characterize the evolution of the cardiac differentiation defects in drugged

395 embryos, we examined the expression of various cardiac differentiation markers at earlier

396 stages of cardiac development (Figure 5). Comparative WISH analyses of BIO- and DMSO-

397 treated embryos showed severely reduced expression of transcription factors regulating

398 cardiovascular development such as $n k x 2.5$, mef2cb, and $s c l$ in response to Wnt

399 overactivation. Consistent with previous studies employing genetic zebrafish models (25), 
400

401

402

403

404

405

406

407

408

expression levels of these genes were dramatically decreased as early as $13 \mathrm{hpf}$ in BIO-treated embryos. Conversely, we observed only mild changes, if any, in the expression of transcription factors with a broader lateral plate mesoderm expression domain, including gata4, gata5, and hand2 (Figure 5). Given that endodermal progenitors of the thyroid lineage develop in close vicinity to the anterior lateral plate mesoderm containing cardiac precursors zebrafish (17), we hypothesized that diminished availability of cardiac mesodermborne signaling cues contributes to the defective thyroid development in BIO-treated embryos.

\section{To evaluate a possible causal relationship between inhibited cardiac development and} thyroid dysgenesis, we examined the co-occurrence of cardiac and thyroid developmental defects over a range of drug concentrations by dual-color WISH of cardiac (myl7) and thyroid ( $n k x 2.4 b)$ marker expression in 28 hpf embryos (Figure 6). For embryos treated with increasing concentrations of BIO from 6 to 10 hpf, we observed a gradual loss of cardiomyocytes correlated with a gradual reduction in thyroid marker expression (Figure 6A-F). Notably, although we observed embryos without any detectable thyroidal $n k x 2.4 b$ expression but still displaying remnant myl7 positive cardiac tissue, we never observed any embryo with detectable thyroid marker expression in the complete absence of myl7 cardiac expression. While these observations are compatible with the hypothesis that the availability of cardiac mesoderm and associated signaling cues are prerequisites for thyroid lineage specification to occur, the concurrent global posteriorization phenotype of the embryos prevented us from distinguishing the relative impact of cardiac dysgenesis against a possible posteriorized patterning in the etiology of the thyroid specification defect.

It has been demonstrated that the later the embryos are treated with Wnt activators during gastrulation, the weaker is the posteriorization effect. Still, cardiac development 
425 remains highly sensitive to these later treatments (25). Therefore, we progressively shifted the

426 treatment periods towards later stages and assessed the impact of drug-induced Wnt signaling

427 on global posterization and cardiac and thyroid development in these embryos. As expected,

428 gross morphological signs of posterization were greatly reduced if embryos were treated from

4299 to 12 hpf (Figure 6G-L). However, Wnt overactivation at these later stages caused still a

430 severe inhibition of cardiac development. Moreover, It appeared that the prevalence and

431 severity of cardiac dysgenesis and thyroid misspecification correlated closely in embryos

432 treated with Wnt-activating drugs between 9 and $12 \mathrm{hpf}$. We made the same observations

433 when we treated the embryos from 10 to $13 \mathrm{hpf}$ or from 9 to $24 \mathrm{hpf}$ (Figure 7). However, we

434 noted that initiation of drug treatment at $11 \mathrm{hpf}$ or later stages caused progressively milder

435 effects on both cardiac and thyroid differentiation as assessed by WISH for cardiac myl7

436 expression and thyroidal $n k x 2.4 b$ expression (Figure 7I,J).

Experimental depletion of cardiomyocyte developmental is associated with thyroid dysgenesis

439 To further examine the hypothesis that depletion of cardiac mesoderm results in a 440 failure of thyroid cell specification, we sought for cardiac differentiation-defective zebrafish

441 models. To the best of our knowledge, a zebrafish model completely lacking cardiac cells

442 while maintaining normal anterior endoderm has not yet been described. Hinits et al. recently

443 described a severe deficiency in cardiomyocyte differentiation in a dual loss-of-function

444 model for zebrafish cardiac transcription factors mef $2 c a$ and mef2cb (51), (52). For our study,

445 we used an antisense morpholino (mef2c/d-MO) developed by the same group that reportedly

446 ablates several Mef2 proteins and faithfully replicates the cardiac phenotype present in

447 mef2calmef $2 c b$ double mutant fish (52). In our hands, injection of the mef $2 c / d$-MO into wild-

448 type embryos, hereafter called Mef2c-deficient embryos, robustly inhibited cardiomyocyte

449 development as evident from only faint myl7 staining of small and very thin heart tube 
450 remnants at 28 and 55 hpf (Figure 8). Compared to control embryos, we also observed a

451 greatly reduced expression of $n k x 2.4 b$ in the thyroid anlage of 28 hpf mef $2 c / d$-morphant

452 embryos (Figure 8A-D). Across several mef2c/d-MO injection experiments, we observed

453 that prevalence and severity of cardiac malformation correlated with the extent of impaired

454 thyroid marker expression in $28 \mathrm{hpf}$ morphant embryos. Detectable domains of $n k x 2.4 b$

455 expression displayed a weaker staining intensity and were of a smaller size in Mef2c-deficient

456 embryos relative to control embryos. Comparison of control and Mef2c-deficient embryos at

$45755 \mathrm{hpf}$ revealed an irregular organization of $t g$-expressing cells in Mef2c-deficient embryos,

458 which displayed a fainter $t g$ staining of individual cells. Collectively, observations made in

459 this Mef2c-deficient zebrafish model of impaired cardiomyocyte differentiation support the

460 contention that thyroid anlage specification relies on normal cardiac development.

Enhancing BMP signaling causes partial rescue of thyroid defects in BIO-treated embryos

463 Cardiac mesoderm expresses a number of diffusible growth factors, including several

464 FGF and BMP ligands (17), (52) with potential thyroid specification-inducing capacity (17),

465 (57), (58). In this respect, our recent small molecule screening showed that pharmacological

466 inhibition of either FGF or BMP signaling during somitogenesis causes a failure of thyroid

467 anlage formation in zebrafish (21). Thus, we reasoned that reduced availability of cardiac-

468 borne signaling cues might explain the defective thyroid anlage formation observed in our

469 experimental models (Wnt overaction, mef2c/d-morphants) displaying perturbed cardiac

470 development in this study.

471 To address the question that diminished BMP signaling might contribute to thyroid 472 phenotypes observed in embryos with after Wnt overactivation, we first examined 473 endogenous bmp4 expression in control embryos and embryos treated with BIO from 6 to 10 474 hpf. WISH analyses of $26 \mathrm{hpf}$ embryos showed that the bmp4 expression of cardiac tissue 
476 figure 5A) is completely ablated in BIO-treated embryos (Supplementary figure 5B).

477 Similarly, Hinits et al. reported that Mef2c-deficient embryos lack bmp4 expression in the 478 cardiac region (52). In normally developing $42 \mathrm{hpf}$ embryos, we also observed that cells 479 contained in the forming thyroid bud display enhanced BMP signaling compared to adjacent 480 foregut tissue (Supplementary figure 5C-E).

481 Next, we used a heat shock-inducible system to globally enhance BMP signaling 482 during somitogenesis stages and assessed the effects of ectopically induced BMP signaling on 483 thyroid development in control and BIO-treated embryos. For this purpose, we treated 484 embryos from $T g(h s p 70 l: b m p 2 b)$ founders with either DMSO $(0.1 \%)$ or BIO $(5 \mu \mathrm{M})$ from 8 485 to $11 \mathrm{hpf}$ and then exposed these embryos to heat-shock treatment at either early 486 somitogenesis (11 hpf), mid-somitogenesis (15 hpf), late somitogenesis (20 hpf) or 487 repeatedly during the course of somitogenesis (at 11, 15, $20 \mathrm{hpf}$ ). Subsequent WISH analyses 488 of thyroid and cardiac markers at 28 hpf revealed different phenotypes depending on the 489 pretreatment (DMSO, BIO) and the timing of heat shock-induced $b m p 2 b$ overexpression.

490 Consistent with a proposed role of BMP for thyroid cell specification, global 491 overactivation of BMP signaling in DMSO-treated embryos (controls) resulted in enhanced 492 expression of $n k x 2.4 b$ (Figure 9A-I). Not only was $n k x 2.4 b$ staining strongly increased in 493 these embryos, but the majority of affected embryos also showed a dramatic and irregular 494 expansion of thyroid marker expression along the anterior-posterior axis. Concurrently, 495 DMSO-treated embryos, when heat-shocked at early somitogenesis, failed to form a normal 496 heart tube but showed a severely perturbed expression pattern of the cardiac marker myl7 with 497 bilateral expression domains extending irregularly along the anterior-posterior axis (Figure 498 9C). When we applied heat-shock on $\operatorname{Tg}(h s p 70 l: b m p 2 b)$ embryos at mid or late somitogenesis 499 stages, we observed that the thyroid anlage and primitive heart tube formed at orthotopic 
500 positions (Figure 9D-G). We also observed a moderate enlargement of the $n k x 2.4 b$

501 expression domain in these embryos. Embryos that were repeatedly heat-shocked during

502 somitogenesis showed a phenotype similar to early somitogenesis heat shock (Figure 9H,I).

503 Collectively, the timed heat-shock experiments in DMSO-treated embryos showed that the

504 endoderm is competent at all somitogenesis stages to respond to BMP overactivation with an

505 enhanced thyroid marker expression.

506 Heat-shock experiments with embryos pretreated with BIO $(5 \mu \mathrm{M})$ from 8-11 hpf

507 showed that global overactivation of BMP signaling could partially rescue the BIO-induced

508 lack of thyroid marker expression at $28 \mathrm{hpf}$. WISH analyses and subsequent genotyping of

509 stained specimens showed that heat-shock treatment of non-transgenic BIO-treated embryos

510 did not improve $n k x 2.4 b$ expression, irrespective of the timing of heat-shock induction during

511 somitogenesis (Figure 9J-R). In stark contrast, BIO-treated embryos carrying the

$512 h s p 70 l: b m p 2 b$ transgene showed a strong $n k x 2.4 b$ expression in the thyroid region when heat-

513 shock induction of BMP signaling was performed at early somitogenesis (11 hpf) (Figure

514 9K,L) or when heat-shock was repeatedly applied at 11, 15 and 20 hpf (Figure 9Q,R). Just

515 as observed in DMSO-treated embryos (see Figure 9C), overactivation of BMP signaling in

516 BIO-treated embryos at early somitogenesis resulted in irregularly shaped and positioned

$517 n k x 2.4 b$ expression domains, often characterized by an elongated shape along the anterior-

518 posterior axis (Figure 9L).

519 The capacity of BMP overactivation to rescue thyroid marker expression in BIO-

520 treated embryos decreased markedly at later somitogenesis stages (see Figure 9N), and no

521 rescue of $n k x 2.4 b$ expression was detectable for heat-shock treatments carried out at $20 \mathrm{hpf}$

522 (see Figure 9P). Thus, in contrast to DMSO-treated embryos, embryos treated with BIO

523 lacked the competence to enhance thyroidal $n k x 2.4 b$ expression in response to overactivation

524 of BMP signaling at late somitogenesis stages. Accordingly, in this model, enhancing BMP 
525 signaling alone at mid- and late somitogenesis stages is not sufficient to rescue thyroid 526 specification defects.

527 One characteristic feature of BIO-treated embryos displaying a rescue in $n k x 2.4 b$ 528 expression following early heat-shock treatment (11 hpf) was the presence of irregular 529 ectopic patches of myl7-expressing cells near the $n k x 2.4 b$-expressing cells. Although we 530 observed variable amounts of myl7-expressing cells across individual embryos from all heat531 shock treatment groups (see Figure 9N,P), it was only in the group of BIO-treated embryos 532 receiving heat-shock treatment at early somitogenesis (11 hpf) that we consistently observed 533 a surplus of myl7-expressing cells in the vicinity of $n k x 2.4 b$-expressing cells. However, we 534 note that we never observed a near full rescue of heart tube formation due to our heat shock treatments in BIO-treated embryos.

Enhancing BMP signaling causes partial rescue of thyroid defects in Mef2c-deficient embryos

538 Given that enhanced BMP signaling could only achieve a rescue of thyroid specification

539 defects in BIO-treated embryos under conditions that concurrently induced a small but 540 consistent cardiac cell differentiation, we next applied a similar rescue approach to Mef2c-

541 deficient. The Mef2c-deficient model provides a promising alternative rescue scenario as 542 global BMP overactivation was deemed unlikely to overcome the block of cardiomyocyte 543 differentiation resulting from the deficiency of mef 2 protein function. In these experiments, 544 we injected embryos from $T g(h s p 70 l: b m p 2 b)$ founders with mef2c/d-MO or maintained $545 T g(h s p 70 l: b m p 2 b)$ embryos as a non-injected control group. Injected and non-injected 546 embryos were then heat-shock treated at early (10 hpf), mid (15 hpf), or late somitogenesis 547 (20 hpf) and thyroid and cardiac markers were analyzed in the different treatment groups at 54828 hpf (Figure 10). In the non-injected control embryos, we observed changes in thyroid and 549 cardiac development (Figure 10A-G) that were very similar to the effects seen in DMSO- 
550 treated control embryos in the previous experimental series (Figure 9A-G). Depending on

551 the timing of heat-shock treatment, we observed ectopic expansions of $n k x 2.4 b$ expression

552 along the anterior-posterior axis (heat-shock at $10 \mathrm{hpf}$ ) or moderately enhanced $n k x 2.4 b$

553 expression when heat-shock was applied at mid (15 hpf) or late somitogenesis stages (20

$554 \mathrm{hpf}$ ). In addition to the irregular shape and position of the thyroidal $n k x 2.4 b$ expression

555 domain, we noted again abnormal myl7 expression domains, irregularly extending along the

556 anterior-posterior axis (Figure 10C).

557 WISH analyses of the thyroid and cardiac marker expression of Mef2c-deficient embryos at $55828 \mathrm{hpf}$ showed a marked reduction in the thyroid $n k x 2.4 b$ staining (Figure 10H). The 559 severity of the thyroid specification defects correlated with the severity of a concurrent 560 cardiac developmental phenotype, as judged from the expression of myl7. As expected, 561 irrespective of their timing, heat-shocks of non-transgenic Mef2c-deficient embryos failed to 562 rescue the thyroid or cardiac phenotype (Figure 10I,K,M). In Mef2c-deficient embryos 563 carrying the hsp70l:bmp2b transgene, heat-shock induction of BMP signaling at early 564 somitogenesis (10 hpf) resulted in a robust thyroid $n k x 2.4 b$ expression, even though the 565 thyroid's shape and position were abnormal (Figure 10J). This phenotype largely resembled 566 the expansion along the anterior-posterior axis seen in control embryos heat-shocked at $10 \mathrm{hpf}$

567 (Figure 10C). Similar to the series of heat-shock experiments performed on BIO-treated 568 embryos, a partial rescue of thyroid specification defects was generally limited to heat-shock 569 induction of BMP signaling at early somitogenesis stages. In Mef2c-deficient embryos 570 carrying the hsp70l:bmp2b transgene, heat-shock induction of BMP signaling at mid- (15 $571 \mathrm{hpf})$ or late somitogenesis (20 hpf) only mildly increased levels of $n k x 2.4 b$ expression 572 compared to untreated mef $2 c / d$ morphants (Figure 10L,N). Compared to the thyroid rescue 573 observed in BIO-treated embryos, a notable difference in the Mef2c-deficient model was that 574 heat-shock induction of BMP signaling did not rescue the cardiac differentiation phenotype. 
bioRxiv preprint doi: https://doi.org/10.1101/2019.12.19.880815; this version posted December 19, 2019. The copyright holder for this preprint (which was not certified by peer review) is the author/funder, who has granted bioRxiv a license to display the preprint in perpetuity. It is made available under aCC-BY-NC-ND 4.0 International license.

575 Thus, our observations in the cardiac deficient mef $2 c / d$ morphant model indicate that

576 ectopically induced BMP signaling is sufficient to rescue the thyroid specification defect even

577 in the absence of cardiomyocyte differentiation. 


\section{Discussion}

579 In this study, we described severe defects in early zebrafish thyroid development as a

580 result of globally modulated canonical Wnt signaling during gastrula and early somitogenesis

581 stages. While thyroid dysgenesis in response to drug-induced Wnt signaling modulation was

582 first detected in the course of our recent small molecule screening study with zebrafish

583 embryos (21), the current study provides corroborative evidence that the thyroid specification

584 defects observed following treatment with BIO and AZA are due to the up-regulation of

585 canonical Wnt signaling. We used a well-characterized zebrafish biosensor line (35) to

586 confirm that BIO and AZA treatments are effectively inducing canonical Wnt signaling in

587 vivo during zebrafish development. Our results are, therefore, in line with numerous studies in

588 zebrafish, Xenopus, and mammalian cell systems utilizing these two compounds to globally

589 induce Wnt signaling (35), (59), (60). Importantly, the thyroid specification defects observed

590 in drugged embryos were faithfully reproduced in a genetic zebrafish, thereby corroborating

591 critical evidence that the thyroid defects are indeed caused by enhanced Wnt signaling and are

592 not due to possible non-specific side effects of drug treatments.

593 Thyroid phenotyping of drugged embryos at the thyroid anlage stage (28 hpf), using

$594 n k x 2.4 b$ expression as a proxy for thyroid lineage commitment, revealed concentration-

595 dependent losses of $n k x 2.4 b$-expressing cells in the prospective thyroid region. In zebrafish

596 embryos, thyroidal expression of $n k x 2.4 b$ typically becomes detectable at around 23/24 hpf,

597 and a global assessment of developmental timing in drugged embryos indicated that reduced

598 detection of $n k x 2.4 b$ expression was not solely due to delayed development. Remarkably,

599 gastrula treatment with a high concentration $(5 \mu \mathrm{M})$ of BIO or AZA caused a complete

600 absence of detectable $n k x 2.4 b$ expression in $28 \mathrm{hpf}$ embryos. Although the latter phenotype

601 was highly penetrant at $28 \mathrm{hpf}$ (affecting almost 100\% of drugged embryos), analyses of

602 treated embryos at later stages (55 hpf) showed that almost all treated embryos developed at 
603 least a very tiny thyroid primordium comprised of very few cells expressing the early

604 functional thyroid marker $\mathrm{tg}$. It is currently unknown if these few remaining $t g$-expressing

605 thyroid cells at $55 \mathrm{hpf}$ are derived from thyroid precursors expressing $n k x 2.4 b$ at such low

606 levels that are undetectable by our WISH at $28 \mathrm{hpf}$ or if some precursor cells are specified

607 after $28 \mathrm{hpf}$ to give rise to $t g$-expressing thyroid cells. Despite the presence of very few

608 differentiated thyroid cells at later stages, we can conclude that increased canonical Wnt

609 signaling during early zebrafish development impairs the specification of thyroid precursor

610 cells.

611 By progressively shifting the timing of short-term drug treatments, we observed that the

612 primary events leading to this thyroid dysgenesis phenotype occur during gastrula and early

613 somitogenesis stages. Therefore, the question arises whether increased Wnt signaling directly

614 affects development of endodermal progenitors, which later give rise to foregut endoderm and

615 the thyroid cell lineage, or whether indirectly through another cell type (e.g. pre-cardiac

616 mesoderm) that is required later in development as a local source of signals to initiate thyroid

617 specification in the foregut endoderm zebrafish (18), (27).

618 During early vertebrate development, Wnt signaling plays a key role in the anteroposterior

619 patterning, mainly by acting as a posteriorizing factor (61). This anterior-posterior patterning

620 role of Wnt signaling has been extensively studied during neural development (55). In our

621 studies, a robust posteriorizing activity of Wnt on neural development was evident from the

622 severe forebrain and eye development defects. Since the thyroid develops from the anterior

623 foregut endoderm, the posteriorizing activity of Wnt on the endoderm could provide a

624 plausible hypothesis to explain the observed thyroid dysgenesis. Surprisingly few studies

625 have addressed the precise role of Wnt on endoderm patterning. Studies in Xenopus embryos

626 indicated that canonical Wnt signaling during gastrulation might pattern the endoderm in a

627 way much similar to what is known for the nervous system (27). Notably, forced Wnt8 
628 expression in cells fated to become endoderm was found to block anterior endoderm

629 development in Xenopus embryos, resulting in specification failure of foregut organ

630 primordia. In contrast to the aforementioned Xenopus studies, morphological and molecular

631 analyses of thyroid-lacking zebrafish embryos revealed the formation of a morphologically

632 fairly normal anterior endoderm with a foregut identity (foxa2 expression) and confirmed

633 timely expression of various other foregut organ markers (hhex, pdx1, proxla). Moreover,

634 data presented by McLin et al. (27) indicate that thyroid specification was preserved in

635 Xenopus embryos if enhanced $\beta$-catenin signaling was cell-autonomously restricted to the

636 endoderm lineage. When comparing these study results with our observations, we conclude

637 that posteriorization effects of BIO-induced Wnt activity were by far not as dramatic as

638 reported in the Xenopus studies and that posteriorization of anterior endoderm patterning was

639 likely not the primary cause for the severe thyroid specification defects in zebrafish embryos.

640

641 Another striking effect of drug-induced Wnt activation was a severe impairment of

642 cardiac differentiation. Consistent with previous studies, we confirmed that enhanced Wnt

643 signaling during zebrafish gastrulation in zebrafish limits cardiac differentiation within pre-

644 cardiac mesoderm (25), (26). Intriguingly, we observed a close correlation between the

645 disruption of cardiac progenitor differentiation and the thyroid abnormalities. This was true

646 for experiments involving increasing concentrations of BIO and AZA as well as for

647 experiments with different treatment periods. In this respect, it is noteworthy that treatment

648 periods, which caused little if any global posteriorization effects, still showed reduced cardiac

649 differentiation concurrent with thyroid specification defects. Thus, the apparent relationship

650 between the diminished formation of cardiac tissue and the corresponding defects in thyroid

651 specification led us to formulate the hypothesis that the primary effect of enhanced Wnt

652 signaling might be the blockage of cardiac differentiation while the thyroid defects might be a 
653 secondary event resulting from a reduction of cardiac-borne signaling cues. This hypothesis is

654 supported by results from several previous studies in different vertebrate models, which

655 provided evidence for a critical role of pre-cardiac and cardiac mesoderm in the induction of

656 thyroid precursor cells signaling (17), (18).

657 Our own studies provided further supporting lines of evidence for this hypothesis by showing 658 that a similar correlation between impaired cardiogenesis and defective thyroid specification 659 exists in Mef2c-deficient embryos (52), a model in which heart formation is blocked 660 independent of perturbations in Wnt signaling. Since the mesoderm, and not the foregut 661 endoderm, expresses mef2ca and mef $2 c b$, this model provided more direct evidence for the 662 contention that thyroid specification relies on proper differentiation of cardiac tissue. A role 663 of mesoderm as a source of signals coming to induce specification of thyroid progenitors 664 within the foregut endoderm is also consistent with what has been already described 665 concerning the specification of other endoderm-derived organs (62), (63), (64).

667 While these observations strongly suggest an important role of cardiac mesoderm as a signal 668 source involved in the early steps of thyroid development, it raises the question of the nature 669 of the signaling cues involved in this tissue-tissue interaction. Based on results from our small 670 molecule screening (21) and previous studies in several other model systems, BMP signaling 671 was deemed a primary candidate of particular importance for thyroid specification (65). In 672 vitro experiments with murine stem cell models identified key conserved roles for BMP 673 signaling in regulating thyroid lineage specification from foregut endoderm (65). On the other 674 hand, Wnt was not required for thyroid specification from endoderm in vitro, like it had been 675 previously suggested (66). Expression profiles during embryogenesis also support a potential 676 role of BMP signaling as a key factor in regulating early steps of thyroid development. In 677 mouse, BMP4 is the Bmp ligand expressed at high levels by the cardiac mesoderm, in 
678 particular within the secondary heart field, the structure that gives rise among others to the

679 outflow tract, which the developing thyroid lies nearby (67), (68).

680 Danesh et al. also demonstrated in mouse embryos that BMPR1a is highly expressed

681 in regions of the pharyngeal endoderm, where endodermal progenitors will differentiate into

682 thyroid cells (68). Thus, Bmp4 could be one critical endogenous Bmp ligand acting from

683 mesoderm to endoderm to initiate thyroid development through interaction with its receptor

684 Bmprla. For zebrafish, we further confirmed a high bmp4 expression in cardiac mesoderm

685 around the time of thyroid specification (Supplementary Figure 5) and ectopic BMP

686 signaling during somitogenesis caused an expansion of the thyroid anlage (Figures $9 \& 10$ ).

687

688 Against this background, it is noteworthy that timed overactivation of BMP signaling during

689 zebrafish somitogenesis stages could partially restore thyroid specification in both models of

690 impaired cardiac development. Interestingly, in our model of cardiac dysgenesis due to

691 enhanced Wnt signaling, the restoration of thyroidal $n k x 2.4 b$ expression achieved by

692 subsequent BMP overactivation appeared strictly dependent on a concurrent rescue of cardiac

693 cell differentiation. At this stage, it is therefore not possible to conclude if thyroid

694 specification was directly restored by the ectopic BMP activity in this model. Several lines of

695 evidence indicate that thyroid specification requires the combined action of BMP and FGF

696 signaling (17), (18), (31), (65), (57). One possible interpretation might be that BMP signaling

697 alone might not be sufficient to induce thyroid specification in embryos with almost no

698 cardiac mesoderm formation. In contrast, restoration to some extent of cardiac differentiation

699 by transient BMP overactivation could provide a source of endogenous BMP, FGF, and

700 possibly other signaling cues to induce thyroid specification.

701 Rescue of thyroid specification was also evident in Mef2c-deficient embryos after BMP

702 overactivation. However, in this model, the correlation between thyroid rescue and the 
703 presence of cardiac mesoderm was less explicit. This correlation reduction was due to the

704 incomplete penetrance of the cardiac phenotype in morphant embryos. However, we observed 705 many Mef2c-deficient embryos presenting a partial thyroid rescue while still lacking 706 cardiomyocytes. These observations indicate that ectopic BMP activation might indeed

707 restore thyroid specification even in the absence of detectable cardiomyocytes. To understand

708 the differences between the thyroid rescue induced by ectopic BMP activity in the two cardiac

709 dysgenesis models, the different mechanisms leading to deficient cardiomyocyte development

710 need to be taken into account. Enhanced Wnt signaling at early stages leads to a block of early

711 cardiac mesoderm differentiation with a pronounced deficit in cardiac progenitor

712 differentiation (see Figure 5). In contrast, studies by Hinits et al (52) showed that early

713 cardiac development is uncompromised in Mef2c-deficient embryos and that the last steps

714 involved in cardiomyocyte differentiation are blocked in this model, including the up-

715 regulation of bmp4 expression in the cardiac field. Thus, pre-cardiac mesoderm present in

716 Mef2c-deficient embryos might serve as a source of FGF and other signaling factors needed

717 to act in concert with BMP to induce thyroid specification. Given that Mef2c-deficient

718 embryos lack bmp4 expression in the cardiac mesoderm and that ectopic overexpression of

$719 m e f 2 c b$ mRNA can induce bmp4 in the cardiac field (52), it would be interesting to

720 investigate further this model, respect to the expression of other potential thyroid-inducing

721 pathways (e.g., FGF) and their role in acting together with BMP signaling in the process of

722 thyroid specification. Also, results obtained in Mef2c-deficient embryos indicate that the

723 thyroid of Bmp4-deficient embryo models might bring further insights into the role of BMP

724 signaling in thyroid specification.

725

726 In summary, our studies confirmed and validated our previous results from a small molecule

727 screen that enhanced Wnt signaling during gastrulation stages results in severe inhibition of 
728 thyroid specification. Moreover, we provide corroborating evidence for the contention that

729 this thyroid dysgenesis results from a blockage of cardiac development and, consequently, the

730 lack of instructive cardiac-derived signaling cues for the specification of thyroid precursor

731 cells. This hypothesis is supported by a similar perturbation of thyroid specification in Mef2c-

732 deficient embryos displaying a lack of cardiomyocyte differentiation. Finally, we demonstrate

733 that ectopic activation of BMP signaling can partially rescue these thyroid phenotypes

734 identifying BMP signaling as one critical component of this tissue-tissue interaction. 


\section{Acknowledgments}

\section{Acknowledgments}

737 We thank J.-M. Vanderwinden from the Light Microscopy Facility for technical assistance.

738 This work was supported by grants from the Belgian National Fund for Scientific Research

739 (FNRS) (FRSM 3-4598-12; CDR-J.0145.16), the Action de Recherche Concertée (ARC) de

740 la Communauté Française de Belgique (ARC AUWB-2012-12/17-ULB3), the Fonds

741 d'Encouragement à la Recherche de l'Université Libre de Bruxelles (FER-ULB), the Fund

742 Yvonne Smits (King Baudouin Fundation) and the Berlin Institute of Health (BIH, CRG-

743 TP2).

744 This work was supported by grant the Belgian National Fund for Scientific Research

745 (FNRS): I.V. is FNRS Research Fellow, B.H. and P.G. are Fund for Research in the Industry

746 and the Agriculture (FRIA) research fellow, R.O. was FNRS Postdoctoral fellow and S.C. is

747 FNRS Senior Research Associate. We greatly appreciate the help of J. Bakkers, E. Moro, S.

748 Abdelilah-Seyfried and G. Weidinger for providing us with transgenic lines used in this study.

749 J. Bakkers for $\operatorname{Tg}(\mathrm{kdrl}: \mathrm{EGFP})$ and $\operatorname{Tg}($ myl7:EGFP) lines, E. Moro for $\operatorname{Tg}(7 x T C F-$

750 Xla.Siam:GFP), S. Abdelilah-Seyfried For Tg(hsp70l:bmp2b) line, G. Weidinger For

$751 \quad \operatorname{Tg}($ hsp70l:wnt8a-EGFP)

752

753 Disclosure Statement

754 The authors have nothing to disclose.

755

756 Corresponding author

757 Please address all correspondences and requests for reprints to: 
758 Sabine Costagliola, Institut de Recherche Interdisciplinaire en Biologie Humaine et

759 Moléculaire, Université Libre de Bruxelles, 808 Route de Lennik, 1070 Brussels, Belgium.

760 Email: $\underline{\text { scostag@ulb.ac.be. }}$

Robert Opitz, Institute of Experimental Pediatric Endocrinology, Charité Universitätsmedizin

Berlin, Augustenburger Platz 1, 13353, Berlin, Germany.

761 Email: robert.opitz@ charite.de.

762

763 References

764 1. De Felice M, Di Lauro R 2004 Thyroid Development and Its Disorders: Genetics

765 and Molecular Mechanisms. Endocrine Reviews 25:722-746.

766 2. Fagman H, Nilsson M 2011 Morphogenetics of early thyroid development. Journal

767 of Molecular Endocrinology 46:R33-R42.

768 3. Deladoey J, Ruel J, Giguere Y, Van Vliet G 2011 Is the incidence of congenital

769

770

771

772

773

774

775

776

777

778

779

780

781

782

783

784

785

786

787

788

789

790

791

792

793

794 hypothyroidism really increasing? A 20-year retrospective population-based study in Quebec. J Clin Endocrinol Metab 96:2422-2429.

4. Vassart G, Dumont JE 2005 Thyroid dysgenesis: multigenic or epigenetic ... or both? Endocrinology 146:5035-5037.

5. Opitz R, Maquet E, Huisken J, Antonica F, Trubiroha A, Pottier G, Janssens V, Costagliola S 2012 Transgenic zebrafish illuminate the dynamics of thyroid morphogenesis and its relationship to cardiovascular development. Dev Biol 372:203-216.

6. Lieschke GJ, Currie PD 2007 Animal models of human disease: zebrafish swim into view. Nature Reviews Genetics 8:353-367.

7. Elsalini OA, Gartzen Jv, Cramer M, Rohr KB 2003 Zebrafish hhex, nk2.1a, and pax2.1 regulate thyroid growth and differentiation downstream of Nodaldependent transcription factors. Dev Biol 263:67-80.

8. Alt B, Reibe S, Feitosa NM, Elsalini OA, Wendl T, Rohr KB 2006 Analysis of origin and growth of the thyroid gland in zebrafish. Developmental dynamics 235:1872-1883.

9. Porazzi P, Calebiro D, Benato F, Tiso N, Persani L 2009 Thyroid gland development and function in the zebrafish model. Molecular and Cellular Endocrinology 312:14-23.

10. Manoli M, Driever W $2014 \mathrm{nkx} 2.1$ and nkx2.4 genes function partially redundant during development of the zebrafish hypothalamus, preoptic region, and pallidum. Front Neuroanat 8:145.

11. De Felice M, Di Lauro R 2011 Minireview: Intrinsic and extrinsic factors in thyroid gland development: an update. Endocrinology 152:2948-2956.

12. Trubiroha A, Gillotay P, Giusti N, Gacquer D, Libert F, Lefort A, Haerlingen B, De Deken X, Opitz R, Costagliola S 2018 A Rapid CRISPR/Cas-based Mutagenesis Assay in Zebrafish for Identification of Genes Involved in Thyroid Morphogenesis $795 \quad$ and Function. Sci Rep 8:5647. 
797 13. Fagman H, Nilsson M 2010 Morphogenesis of the thyroid gland. Mol Cell

798

799

800

801

802

803

804

805

806

807

808

809

810

811

812

813

814

815

816

817

818

819

820

821

822

823

824

825

826

827

828

829

830

831

832

833

834

835

836

837

838

839

840

841

842

843

844

845 Endocrinol 323:35-54.

14. Alt B, Elsalini OA, Schrumpf P, Haufs N, Lawson N, Schwabe G, Mundlos S, Grüters A, Krude H, Rohr KB 2006 Arteries define the position of the thyroid gland during its developmental relocalisation. Development 133.

15. Opitz R, Hitz MP, Vandernoot I, Trubiroha A, Abu-Khudir R, Samuels M, Desilets V, Costagliola S, Andelfinger G, Deladoey J 2015 Functional zebrafish studies based on human genotyping point to netrin-1 as a link between aberrant cardiovascular development and thyroid dysgenesis. Endocrinology 156:377-388.

16. Olivieri A, Stazi M, Mastroiacovo P, Fazzini C, Medda E, Spagnolo A, De Angelis S, Grandolfo M, Taruscio D, Cordeddu V, Sorcini M, Hypothyroidism SGfC 2002 A population-based study on the frequency of additional congenital malformations in infants with congenital hypothyroidism: data from the Italian Registry for Congenital Hypothyroidism (1991-1998). Journal of Clinical Endocrinology and Metabolism 87:557-562.

17. Wendl T, Adzic D, Schoenebeck J, Scholpp S, Brand M, Yelon D, Rohr K 2007 Early developmental specification of the thyroid gland depends on han-expressing surrounding tissue and on FGF signals. Development 134:2871-2879.

18. Lania G, Zhang Z, Huynh T, Caprio C, Moon AM, Vitelli F, Baldini A 2009 Early thyroid development requires a Tbx1-Fgf8 pathway. Dev Biol 328:109-117.

19. Porazzi P, Marelli F, Benato F, de Filippis T, Calebiro D, Argenton F, Tiso N, Persani L 2012 Disruptions of global and jagged1-mediated notch signaling affect thyroid morphogenesis in the zebrafish. Endocrinology 153:5645-5658.

20. Stainier DY 2001 Zebrafish genetics and vertebrate heart formation. Nature reviews 2:39-48.

21. Haerlingen B, Opitz R, Vandernoot I, Trubiroha A, Gillotay P, Giusti N, Costagliola S 2019 Small-Molecule Screening in Zebrafish Embryos Identifies Signaling Pathways Regulating Early Thyroid Development. Thyroid.

22. Steinhart Z, Angers S $2018 \mathrm{Wnt}$ signaling in development and tissue homeostasis. Development 145.

23. He X, Semenov M, Tamai K, Zeng X 2004 LDL receptor-related proteins 5 and 6 in Wnt/beta-catenin signaling: arrows point the way. Development 131:1663-1677.

24. Gordon MD, Nusse R 2006 Wnt signaling: multiple pathways, multiple receptors, and multiple transcription factors. J Biol Chem 281:22429-22433.

25. Dohn TE, Waxman JS 2012 Distinct phases of Wnt/beta-catenin signaling direct cardiomyocyte formation in zebrafish. Dev Biol 361:364-376.

26. Ueno S, Weidinger G, Osugi T, Kohn AD, Golob JL, Pabon L, Reinecke H, Moon RT, Murry CE 2007 Biphasic role for Wnt/beta-catenin signaling in cardiac specification in zebrafish and embryonic stem cells. Proc Natl Acad Sci U S A 104:9685-9690.

27. McLin VA, Rankin SA, Zorn AM 2007 Repression of Wnt/beta-catenin signaling in the anterior endoderm is essential for liver and pancreas development. Development 134:2207-2217.

28. Poulain M, Ober EA 2011 Interplay between Wnt2 and Wnt2bb controls multiple steps of early foregut-derived organ development. Development 138:3557-3568.

29. Westerfield M 2000 The zebrafish book. A guide for the laboratory use of zebrafish (Danio rerio). University of Oregon press 4th ed. Eugene, OR.

30. Kimmel CBea 1995 Stages of embryonic development of the zebrafish. Developmental dynamics 203:253-310. 
846

31. Longmire TA, Ikonomou L, Hawkins F, Christodoulou C, Cao Y, Jean JC, Kwok LW, Mou H, Rajagopal J, Shen SS, Dowton AA, Serra M, Weiss DJ, Green MD, Snoeck HW, Ramirez MI, Kotton DN 2012 Efficient derivation of purified lung and thyroid progenitors from embryonic stem cells. Cell Stem Cell 10:398-411.

32. White R, Sessa A, Burke C, Bowman T, LeBlanc J, Ceol C, Bourque C, Dovey M, Goessling W, Burns C, Zon L 2008 Transparent adult zebrafish as a tool for in vivo transplantation analysis. Cell Stem Cell 2:183-189.

33. Jin SW, Herzog W, Santoro MM, Mitchell TS, Frantsve J, Jungblut B, Beis D, Scott IC, D'Amico LA, Ober EA, Verkade H, Field HA, Chi NC, Wehman AM, Baier H, Stainier DY 2007 A transgene-assisted genetic screen identifies essential regulators of vascular development in vertebrate embryos. Dev Biol 307:29-42.

34. Huang CJ, Tu CT, Hsiao CD, Hsieh FJ, Tsai HJ 2003 Germ-line transmission of a myocardium-specific GFP transgene reveals critical regulatory elements in the cardiac myosin light chain 2 promoter of zebrafish. Dev Dyn 228:30-40.

35. Moro E, Ozhan-Kizil G, Mongera A, Beis D, Wierzbicki C, Young RM, Bournele D, Domenichini A, Valdivia LE, Lum L, Chen C, Amatruda JF, Tiso N, Weidinger G, Argenton F 2012 In vivo Wnt signaling tracing through a transgenic biosensor fish reveals novel activity domains. Developmental Biology 366:327-340.

36. Mizoguchi T, Verkade H, Heath JK, Kuroiwa A, Kikuchi Y 2008 Sdf1/Cxcr4 signaling controls the dorsal migration of endodermal cells during zebrafish gastrulation. Development 135:2521-2529.

37. Chocron S, Verhoeven MC, Rentzsch F, Hammerschmidt M, Bakkers J 2007 Zebrafish Bmp4 regulates left-right asymmetry at two distinct developmental time points. Dev Biol 305:577-588.

38. Weidinger G, Thorpe C, Wuennenberg-Stapleton K, Ngai J, Moon R 2005 The Sp1related transcription factors sp5 and sp5-like act downstream of Wnt/betacatenin signaling in mesoderm and neurectoderm patterning. Current Biology 29:489-500.

39. Karlsson J, Hofsten Jv, Olsson P-E 2001 Generating Transparent Zebrafish: A Refined Method to Improve Detection of Gene Expression During Embryonic Development. Marine Biotechnology 3:522-527.

40. Meijer L, Flajolet M, Greengard P 2004 Pharmacological inhibitors of glycogen synthase kinase 3. Trends Pharmacol Sci 25:471-480.

41. Chen B, Dodge ME, Tang W, Lu J, Ma Z, Fan CW, Wei S, Hao W, Kilgore J, Williams NS, Roth MG, Amatruda JF, Chen C, Lum L 2009 Small molecule-mediated disruption of Wnt-dependent signaling in tissue regeneration and cancer. Nat Chem Biol 5:100-107.

42. Shin D, Shin CH, Tucker J, Ober EA, Rentzsch F, Poss KD, Hammerschmidt M, Mullins MC, Stainier DY 2007 Bmp and Fgf signaling are essential for liver specification in zebrafish. Development 134:2041-2050.

43. Yelon D, Horne S, Stainier D 1999 Restricted expression of cardiac myosin genes reveals regulated aspects of heart tube assembly in zebrafish. Dev Biol 214:2337.

44. Yelon D, Ticho B, Halpern M, Ruvinsky I, Ro R, Silver L, Stainier D 2000 The bHLH transcription factor hand2 plays parallel roles in zebrafish heart and pectoral fin development. Development 127:2573-2582.

45. Liao E, Paw B, Oates A, Pratt S, Postlethwait J, Zon L 1998 SCL/Tal-1 transcription factor acts downstream of cloche to specify hematopoietic and vascular progenitors in zebrafish. Genes Development 12:621-626. 
895 46. Thisse CT, B. 2008 High-resolution in situ hybridization to whole-mount zebrafish embryos. Nat Protoc 3:59-69.

897

898

899

900

901

902

903

904

905

906

907

908

909

910

911

912

913

914

915

916

917

918

919

920

921

922

923

924

925

926

927

928

929

930

931

932

933

934

935

936

937

938

939

940

941

47. Opitz R, Maquet E, Zoenen M, Dadhich R, Costagliola S 2011 TSH Receptor Function Is Required for Normal Thyroid Differentiation in Zebrafish. Molecular Endocrinolgy 25:1579-1599.

48. Dorsky RI, Sheldahl LC, Moon RT 2002 A transgenic Lef1/beta-catenindependent reporter is expressed in spatially restricted domains throughout zebrafish development. Dev Biol 241:229-237.

49. Pfaffl M 2001 A new mathematical model for relative quantification in real-time RT-PCR. Nucleic Acids Research 29:e45.

50. Tang R, Dodd A, Lai D, McNabb W, Love D 2007 Validation of zebrafish (Danio rerio) reference genes for quantitative real-time RT-PCR normalization. Acta Biochim Biophys Sin (Shangai) 39:384-390.

51. Hinits $Y$, Hughes SM 2007 Mef2s are required for thick filament formation in nascent muscle fibres. Development 134:2511-2519.

52. Hinits Y, Pan L, Walker C, Dowd J, Moens CB, Hughes SM 2012 Zebrafish Mef2ca and Mef2cb are essential for both first and second heart field cardiomyocyte differentiation. Dev Biol 369:199-210.

53. van de Water S, van de Wetering M, Joore J, Esseling J, Bink R, Clevers H, Zivkovic D 2001 Ectopic Wnt signal determines the eyeless phenotype of zebrafish masterblind mutant. Development 128:3877-3888.

54. Yamaguchi TP 2001 Heads or tails: Wnts and anterior-posterior patterning. Curr Biol 11:R713-724.

55. Green D, Whitener A, Mohanty S, Lekven A 2015 Vertebrate nervous system posteriorization: Grading the function of Wnt signaling. Dev Dyn 244:507-512.

56. Manfroid I, Delporte F, Baudhuin A, Motte P, Neumann CJ, Voz ML, Martial JA, Peers B 2007 Reciprocal endoderm-mesoderm interactions mediated by fgf 24 and fgf10 govern pancreas development. Development 134:4011-4021.

57. Serra M, Alysandratos KD, Hawkins F, McCauley KB, Jacob A, Choi J, Caballero IS, Vedaie M, Kurmann AA, Ikonomou L, Hollenberg AN, Shannon JM, Kotton DN 2017 Pluripotent stem cell differentiation reveals distinct developmental pathways regulating lung- versus thyroid-lineage specification. Development 144:3879-3893.

58. Kurmann Anita A, Serra M, Hawkins F, Rankin Scott A, Mori M, Astapova I, Ullas S, Lin S, Bilodeau M, Rossant J, Jean Jyh C, Ikonomou L, Deterding Robin R, Shannon John M, Zorn Aaron M, Hollenberg Anthony N, Kotton Darrell N 2015 Regeneration of Thyroid Function by Transplantation of Differentiated Pluripotent Stem Cells. Cell Stem Cell 17:527-542.

59. Chen EY, DeRan MT, Ignatius MS, Grandinetti KB, Clagg R, McCarthy KM, Lobbardi RM, Brockmann J, Keller C, Wu X, Langenau DM 2014 Glycogen synthase kinase 3 inhibitors induce the canonical WNT/ -catenin pathway to suppress growth and self-renewal in embryonal rhabdomyosarcoma. Proceedings of the National Academy of Sciences 111:5349-5354.

60. Meijer L, Skaltsounis A-L, Magiatis P, Polychronopoulos P, Knockaert M, Leost M, Ryan XP, Vonica CA, Brivanlou A, Dajani R, Crovace C, Tarricone C, Musacchio A, Roe SM, Pearl L, Greengard P 2003 GSK-3-Selective Inhibitors Derived from Tyrian Purple Indirubins. Chemistry \& Biology 10:1255-1266. 
942 61. Tuazon FB, Mullins MC 2015 Temporally coordinated signals progressively

943

944

945

946

947

948

949

950

951

952

953

954

955

956

957

958

959

960

961

962

963

964

965

966

967

968

969

970

971

972

973

974

975

976

977

978

979 pattern the anteroposterior and dorsoventral body axes. Semin Cell Dev Biol 42:118-133.

62. Deutsch G, Jung J, Zheng M, Lora J, Zaret KS 2001 A bipotential precursor population for pancreas and liver within the embryonic endoderm. Development 128:871-881.

63. Ober EA, Verkade H, Field HA, Stainier DY 2006 Mesodermal Wnt2b signalling positively regulates liver specification. Nature 442:688-691.

64. Neves H, Dupin E, Parreira L, Le Douarin NM 2012 Modulation of Bmp4 signalling in the epithelial-mesenchymal interactions that take place in early thymus and parathyroid development in avian embryos. Dev Biol 361:208-219.

65. Kurmann AA, Serra M, Hawkins F, Rankin SA, Mori M, Astapova I, Ullas S, Lin S, Bilodeau M, Rossant J, Jean JC, Ikonomou L, Deterding RR, Shannon JM, Zorn AM, Hollenberg AN, Kotton DN 2015 Regeneration of Thyroid Function by Transplantation of Differentiated Pluripotent Stem Cells. Cell Stem Cell 17:527542.

66. Goss AM, Tian Y, Tsukiyama T, Cohen ED, Zhou D, Lu MM, Yamaguchi TP, Morrisey EE $2009 \mathrm{Wnt} 2 / 2 \mathrm{~b}$ and beta-catenin signaling are necessary and sufficient to specify lung progenitors in the foregut. Dev Cell 17:290-298.

67. Klaus A, Saga Y, Taketo MM, Tzahor E, Birchmeier W 2007 Distinct roles of Wnt/beta-catenin and Bmp signaling during early cardiogenesis. Proceedings of the National Academy of Sciences of the United States of America 104:1853118536.

68. Danesh SM, Villasenor A, Chong D, Soukup C, Cleaver O 2009 BMP and BMP receptor expression during murine organogenesis. Gene Expr Patterns 9:255265.

\section{Figure Legends}

Fig. 1. Drug-induced overactivation of canonical Wnt signaling during gastrulation impairs thyroid specification. (A-H) Expression of the early thyroid marker $n k x 2.4 b$ in 28 hpf embryos following treatment with $0.1 \%$ DMSO (vehicle control) and two Wnt-inducing drugs, BIO and azakenpaullone (AZA) between 6 and 10 hpf. In control embryos (A,B,E,F), $n k x 2.4 b$ is expressed in the thyroid anlage (T) and the ventral forebrain (hypothalamus, $\mathrm{H}$ ). In response to $\mathrm{BIO}(\mathrm{C}-\mathrm{D})$ and $\mathrm{AZA}(\mathrm{G}-\mathrm{H})$ treatment, $n k x 2.4 b$ mRNA expression is lost in both domains in the majority of embryos. Arrows point to $n k x 2.4 b$ expression in the thyroid anlage. Dorsal views with anterior oriented to the top (A,C,E,G) and lateral views with anterior oriented to the left $(\mathrm{B}, \mathrm{D}, \mathrm{F}, \mathrm{H})$ are shown. (I): Quantification of the proportion of 
980 specimen displaying thyroid specification defects following AZA treatment, as determined by

$981 n k x 2.4 b$ staining of $28 \mathrm{hpf}$ embryos. Results are presented as the percentage of embryos

982 displaying a particular phenotype, showing a concentration-dependent $n k x 2.4 b$ mRNA loss of

983 expression. The total number of specimens analyzed for each treatment group is provided on

984 the top of each column. Scale bar, $200 \mu \mathrm{m}$.

985

986 Fig. 2. Drug-induced overactivation of canonical Wnt signaling during gastrulation

987 impairs the thyroid primordium formation. (A-H) Expression of the thyroid marker

$988 n k x 2.4 b$ in 55 hpf embryos following treatment with $0.1 \%$ DMSO (vehicle control) and the

989 Wnt-inducing drug BIO between 6 and 10 hpf. In control embryos (A,E), $n k x 2.4 b$ is

990 expressed in the thyroid primordium (T) and the ventral forebrain (hypothalamus, $\mathrm{H}$ ). In

991 response to $\mathrm{BIO}(\mathrm{B}-\mathrm{D}, \mathrm{F}-\mathrm{H})$ treatment, $n k x 2.4 b$ mRNA expression is reduced or lost in both

992 tissues in a concentration-dependent manner. Arrows point to $n k x 2.4 b$ expression in the

993 thyroid primordium. Lateral views with anterior oriented to the left (A-D) and dorsal views

994 with anterior oriented to the top (E-H) are shown. (I): Quantification of the percentage of

995 embryos displaying a particular phenotype following BIO treatment, as determined by

$996 n k x 2.4 b$ staining of $55 \mathrm{hpf}$ embryos. Total numbers of specimens analyzed for each treatment

997 group are provided on the top of each column. (J-Q) Expression of the terminal thyroid

998 differentiation marker $t g$ in 55 hpf embryos following treatment with $0.1 \%$ DMSO (vehicle

999 control) and the Wnt-inducing drug BIO between 6 and 10 hpf. In control embryos (J,N), $t g$

1000 is exclusively expressed in the thyroid primordium (T). In response to BIO treatment (K-

$1001 \mathrm{M}, \mathrm{O}-\mathrm{Q}), \operatorname{tg}$ mRNA expression is reduced or lost in a concentration-dependent manner.

1002 Arrows point to the thyroid primordium. Lateral views with anterior oriented to the left (J-M)

1003 and dorsal views with anterior oriented to the top (N-Q) are shown. (I): Quantification of the

1004 percentage of embryos displaying a particular phenotype following BIO treatment, as 
1005 determined by $t g$ staining of 55 hpf embryos. Total numbers of specimens analyzed for each

1006 treatment group are provided on the top of each column. Scale bar, $200 \mu \mathrm{m}$.

1007

1008 Fig. 3. Heat shock-induced overexpression of wnt8a-GFP during gastrulation impairs

1009 thyroid specification and thyroid primordium formation.

1010 (A,B) Expression of the thyroid marker $n k x 2.4 b$ in 28 hpf embryos following heat shock

1011 (HS) treatment of $T g($ hsp70l:wnt8a-GFP) embryos at 6 hpf. In response to HS, embryos

1012 carrying the HS-inducible wnt8a-GFP transgene showed a great reduction or even loss of

$1013 n k x 2.4 b$ mRNA expression in the thyroid anlage (T) and the ventral forebrain

1014 (hypothalamus, H), whereas expression of $n k x 2.4 b$ in both tissues was unaffected after HS of

1015 non-transgenic siblings. Dorsal views with anterior oriented to the top are shown. Arrows

1016 point to $n k x 2.4 b$ expression in the thyroid anlage. (C,D) Expression of the terminal thyroid

1017 differentiation marker $\mathrm{tg}$ in $55 \mathrm{hpf}$ embryos following heat shock (HS) treatment of

$1018 T g(h s p 70 l: w n t 8 a-G F P)$ embryos at 6 hpf. Note the strongly reduced size of the thyroid

1019 primordium in embryos carrying the HS-inducible wnt $8 a-G F P$ transgene compared to normal

1020 thyroid size in non-transgenic siblings. Similar to drug-induced wnt overactivation, HS-

1021 induced overexpression of the wnt8a-GFP transgene resulted in a dramatic neural

1022 posteriorization phenotype (loss of anterior neural tissue, lack of eyes). Ventral views with

1023 anterior oriented to the top are shown. Arrows point to $t g$ expression in the thyroid

1024 primordium. Scale bar, $200 \mu \mathrm{m}$.

1025

1026 Fig. 4. Overactivation of canonical Wnt signaling during gastrula stages has limited

1027 effects on markers of endodermal patterning and endodermal organogenesis. (A-L)

1028 Comparative whole-mount in situ hybridization analysis of mRNA expression patterns for a

1029 panel of markers of endodermal patterning in embryos treated with $0.1 \%$ DMSO (vehicle 
1030 control) and $5 \mu \mathrm{M}$ BIO from $6-10$ hpf. In contrast to the lack of $n k x 2.4 b \quad(\mathbf{A , B})$ and hhex

1031 (C,D) expression in the region of the thyroid anlage (T) of BIO-treated embryos, hhex

1032 expression is maintained in the region of the prospective liver $(\mathrm{L})$ and pancreas $(\mathrm{P})$

1033 development. Robust foxa2 expression (E,F) in BIO-treated embryos indicates that

1034 specification of foregut endoderm (FE) was not blocked by Wnt overactivation. However,

1035 expression of the mid-/hindgut marker foxa3 (G,H) was expanded towards more anterior

1036 regions in several BIO-treated embryos suggesting possible posteriorization effects on

1037 endodermal patterning. Importantly, BIO-treated embryos displayed robust staining for all

1038 markers of hepatic and pancreatic development including hhex (C,D), foxa3 (G,H), pdx1

$1039(\mathbf{I}, \mathbf{J})$ and proxla $(\mathbf{K}, \mathbf{L})$ while showing a specific reduction or lack of markers of early thyroid

1040 development. Long arrows point to the thyroid, short arrows to the liver, and arrowheads to

1041 pancreatic domains. Brackets in panels E,F demarcate the foregut endoderm (FE) domain of

1042 foxa2 expression. Panels A-J show dorsal views with anterior oriented to the top. Panels K

1043 and L show lateral views with anterior oriented to the left. Scale bar, $200 \mu \mathrm{m}$. 
1045 Fig. 5. Drug-induced overactivation of canonical Wnt signaling during gastrulation

1046 impairs early stages of cardiac development. (A-L) Whole-mount in situ hybridization

1047 analysis of mRNA expression for a panel of cardiac differentiation markers in 13 hpf embryos

1048 treated with $0.1 \%$ DMSO (vehicle control) and $5 \mu \mathrm{M}$ BIO from $6-10$ hpf. BIO treatment

1049 severely blocked early stages of cardiac development, as shown by the dramatic reduction in

1050 early cardiac markers $n k x 2.5(\mathbf{A}, \mathbf{B})$ and mef2cb $(\mathbf{C , D})$. In addition, there is a severe

1051 impairment of endothelial precursor formation, as indicated by the loss of $s c l$ expression

1052 (E,F) in BIO-treated embryos. Wnt overactivation specifically affected cardiac and

1053 endothelial precursor development, as shown by robust expression of other markers of lateral

1054 plate mesoderm development including hand2 (G,H), gata4 (I,J), and gata5 (K,L). Dorsal

1055 views with anterior oriented to the top are shown. (M-P) Expression analysis of the

1056 myocardial marker myl7 at 19 hpf reveals concentration-dependent effects of early BIO

1057 treatment on heart morphogenesis, as evident from the impaired cardiac cone formation,

1058 delayed midline fusion of bilateral cardiac fields, and severe depletion of differentiated

1059 cardiomyocytes. Arrows point to myl7 expression in the region of the forming cardiac cone.

1060 Dorsal views with anterior oriented to the top are shown. Scale bar, $200 \mu \mathrm{m}$.

1061

1062 Fig. 6. Thyroid specification defects coincide with impaired cardiac development in 1063 embryos following drug-induced overactivation of canonical Wnt signaling. (A-F) Dual-

1064 color whole-mount in situ hybridization analysis of mRNA expression of markers for early

1065 thyroid $(n k x 2.4 b)$ and cardiac (myl7) development in 28 hpf embryos treated with $0.1 \%$

1066 DMSO (vehicle control) and varying concentrations of BIO from 6-10 hpf. Wnt

1067 overactivation during this period concurrently affected thyroid and cardiac development

1068 though thyroid specification appeared slightly more sensitive to BIO treatment. The severity

1069 of the thyroid defects was tightly correlated to the degree of neural posteriorization effects. 
1070 (G-L) Dual-color in situ hybridization analysis of $n k x 2.4 b$ and $m y l 7$ expression in 28 hpf

1071 embryos treated with $0.1 \%$ DMSO (vehicle control) and varying concentrations of

1072 azakenpaullone (AZA) from 9-12 hpf. Wnt overactivation during this slightly later period

1073 resulted in few if any neural posteriorization phenotypes, whereas thyroid and cardiac

1074 development were concurrently affected, showing a similar sensitivity to perturbed Wnt

1075 signaling. Dorsal views (A,C,E,G,I,K) with anterior to the top and lateral views

1076 (B,D,F,H,J,L) with anterior to the left are shown. Blue arrows point to thyroidal $n k x 2.4 b$

1077 expression and red arrows to myl7 expression in the heart tube. Scale bar, $200 \mu \mathrm{m}$

1078

1079 Fig. 7. Time-dependent effects of Wnt overactivation on thyroid and cardiac 1080 development during gastrulation and early somitogenesis stages.

1081 (A-L) Dual-color whole-mount in situ hybridization analysis of mRNA expression of 1082 markers for early thyroid $(n k x 2.4 b)$ and cardiac (myl7) development in 28 hpf embryos 1083 treated with $0.1 \%$ DMSO (vehicle control) and azakenpaullone (AZA) during different 1084 periods of early development. Note that neural posteriorization phenotypes became much less 1085 prominent and severe when drug-induced Wnt overactivation was initiated at late gastrula 1086 stages. Concurrent defects in thyroid and cardiac development were detected for all drug 1087 treatments that started before $11 \mathrm{hpf}$. Prevalence and severity of thyroid and cardiac 1088 phenotypes showed a close correlation across different treatment periods. Blue arrows point 1089 to thyroidal $n k x 2.4 b$ expression and red arrows to myl7 expression in the heart tube. Dorsal 1090 views (A,C,E,G,I,K) with anterior to the top and lateral views (B,D,F,H,J,L) with anterior 1091 to the left are shown. Scale bar, $200 \mu \mathrm{m}$. (M,N) Quantification of the proportion of specimen 1092 displaying defects in thyroid specification (M) and cardiac development (N) following AZA 1093 treatment, as determined by $n k x 2.4 b$ (M) and myl7 (N) staining of $28 \mathrm{hpf}$ embryos. Results 1094 are presented as the percentage of embryos displaying a particular phenotype. The total 
1095 number of specimens analyzed for each treatment group is provided on the top of each

1096 column.

1097

1098 Fig. 8. Impaired cardiac development in mef2c/d morpholino-injected embryos is 1099 associated with reduced thyroid marker expression.

1100 (A-D) Dual-color whole-mount in situ hybridization analysis of mRNA expression of 1101 markers for early thyroid $(n k x 2.4 b)$ and cardiac (myl7) development in 28 hpf embryos 1102 injected with mef2c/d morpholino (MO) and non-injected control embryos (NI). MO 1103 injection caused variable loss of differentiated cardiomyocytes and resulted in smaller hearts

1104 (C) compared to controls. Concurrently, these embryos displayed reduced staining of $n k x 2.4 b$

1105 (C). (E-H) Dual-color whole-mount in situ hybridization analysis of mRNA expression of 1106 myl7 and the thyroid differentiation marker $t g$ in $55 \mathrm{hpf}$ embryos injected with mef2c/d 1107 morpholino (MO) and non-injected control embryos (NI). MO-injected embryos displayed 1108 small, string-like midline hearts $(\mathbf{G})$ and a modest reduction in $t g$ staining (G). Blue arrows 1109 point to thyroidal $n k x 2.4 b$ and $t g$ expression and red arrows to $m y l 7$ expression in the heart 1110 tube. Dorsal views $(\mathbf{A}, \mathbf{C})$ with anterior to the top, lateral views $(\mathbf{B}, \mathbf{D}, \mathbf{F}, \mathbf{H})$ with anterior to 1111 the left and ventral views $(\mathbf{E}, \mathbf{G})$ with anterior to the top are shown. Scale bar, $200 \mu \mathrm{m}$.

1113 Fig. 9. Conditional overactivation of BMP signaling in $\operatorname{Tg}($ hsp70l:bmp2b) embryos 1114 partially rescues BIO-induced thyroid specification defects. (A-R) Dual-color whole1115 mount in situ hybridization analysis of thyroid (nkx2.4b) and cardiac (myl7) markers in 28 1116 hpf embryos carrying the HS-inducible $b m p 2 b$ transgene $(b m p 2 b+)$ and in non-transgenic 1117 siblings (bmp2b-). Panels A-I show results for embryos that were treated with 0.1\% DMSO 1118 (vehicle control) from 8-11 hpf and were subsequently exposed to timed heat shock (HS) 1119 treatment at early (11 hpf), mid (15 hpf) or late somitogenesis (20 hpf) and for embryos 
1120 exposed to repeated HS at 11,15 and $20 \mathrm{hpf}$. Carriers of the $b m p 2 b$ transgene showed

1121 enhanced $n k x 2.4 b$ expression in response to HS irrespective of the specific timing of HS

1122 treatment. Note that HS at $11 \mathrm{hpf}(\mathbf{B}, \mathbf{C})$ and repeated HS treatment $(\mathbf{H , I})$ disrupted heart

1123 formation and caused ectopic stretches of myl7 expression bilateral to the midline. Panels J-R

1124 show corresponding effects of HS treatment for embryos that were treated with $5 \mu \mathrm{M}$ BIO

1125 from 8-11 hpf. HS of non-trangenic embryos did not affect the BIO-induced deficits in

1126 thyroid and cardiac development (compare $\mathbf{J}$ to $\mathbf{K}, \mathbf{M}, \mathbf{O}, \mathbf{Q}$ ). Carriers of the $b m p 2 b$ transgene

1127 showed variable levels of $n k x 2.4 b$ expression rescue, depending on the specific timing of HS

1128 treatment. Note that HS at $11 \mathrm{hpf}(\mathbf{K}, \mathbf{L})$ and the repeated HS treatment $(\mathbf{Q}, \mathbf{R})$ were most

1129 effective in restoring robust $n k x 2.4 b$ expression. Partial recovery of myl7 expression was

1130 detectable in response to $b m p b 2 b$ overexpression, but heart tube formation was never

1131 restored. Dorsal views with anterior oriented to the top are shown. Blue arrows point to

$1132 n k x 2.4 b$ expression in the thyroid anlage and red arrows to domains of $m y l 7$ expression. Scale

1133 bar: $200 \mu \mathrm{m}$. (S,T) Quantification of the proportion of specimens displaying thyroid (S) and

1134 cardiac phenotypes (T) in response to different HS treatments as determined by $n k x 2.4 b$ (S)

1135 and myl7 (T) staining of $28 \mathrm{hpf}$ embryos. Results are presented as the percentage of embryos

1136 displaying a particular phenotype. The total number of specimens analyzed for each treatment

1137 group is provided on the top of each column.

1139 Fig. 10. Conditional overactivation of BMP signaling in $\operatorname{Tg}(h \operatorname{sp} 70 l: b m p 2 b)$ embryos 1140 partially rescues mef2c/d morpholino-induced thyroid specification defects.

1141 (A-N) Dual-color whole-mount in situ hybridization analysis of thyroid $(n k x 2.4 b)$ and 1142 cardiac (myl7) markers in $28 \mathrm{hpf}$ embryos carrying the HS-inducible bmp2b transgene 1143 (bmp2b+) and in non-transgenic siblings (bmp2b-). Panels A-G show results for control 1144 embryos exposed to timed heat shock (HS) treatment at early (10 hpf), mid (15 hpf) or late 
1145 somitogenesis (20 hpf). Carriers of the $b m p 2 b$ transgene showed enhanced $n k x 2.4 b$

1146 expression in response to HS irrespective of the specific timing of HS treatment. Note that HS

1147 at $10 \mathrm{hpf}(\mathbf{B}, \mathbf{C})$ disrupted heart formation and caused ectopic stretches of myl7 expression

1148 bilateral to the midline. Panels H-N show corresponding effects of HS treatment for embryos

1149 that were injected with mef2c/d morpholino (MO) at the one-cell stage. HS of non-trangenic

1150 embryos did not affect the MO-induced deficits in thyroid and cardiac development (compare

$1151 \mathbf{H}$ to $\mathbf{I , K}, \mathbf{M})$. Carriers of the $b m p 2 b$ transgene showed variable levels of $n k x 2.4 b$ expression

1152 rescue, depending on the specific timing of HS treatment. Note that HS at $10 \mathrm{hpf}$ (I, J) was

1153 most effective in restoring robust $n k x 2.4 b$ expression. No significant recovery of myl7

1154 expression was detectable in response to $b m p b 2 b$ overexpression. Dorsal views with anterior

1155 oriented to the top are shown. Blue arrows point to $n k x 2.4 b$ expression in the thyroid anlage

1156 and red arrows to domains of myl7 expression. Scale bar: $200 \mu \mathrm{m}$. (O,P) Quantification of

1157 the proportion of specimens displaying thyroid $(\mathbf{O})$ and cardiac phenotypes $(\mathbf{P})$ in response

1158 to different HS treatments as determined by $n k x 2.4 b \quad(\mathbf{O})$ and myl7 $(\mathbf{P})$ staining of $28 \mathrm{hpf}$

1159 embryos. Results are presented as the percentage of embryos displaying a particular

1160 phenotype. The total number of specimens analyzed for each treatment group is provided on

1161 the top of each column.

1162

1163 


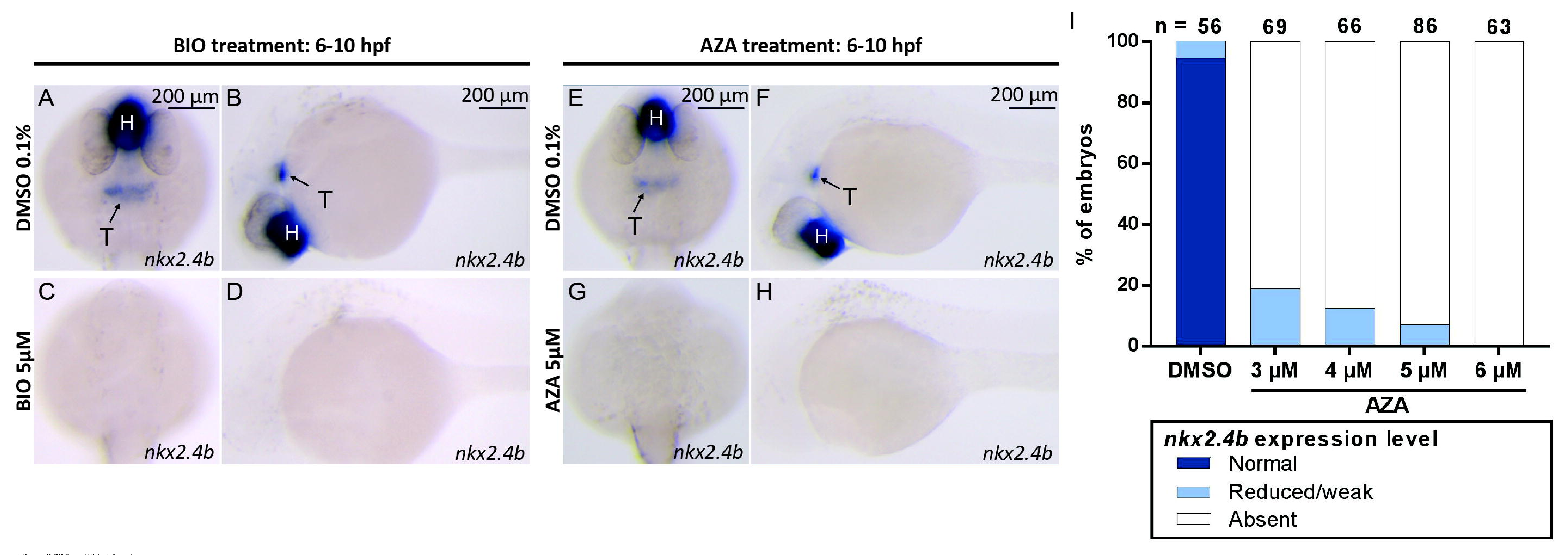


BIO treatment: 6-10 hp

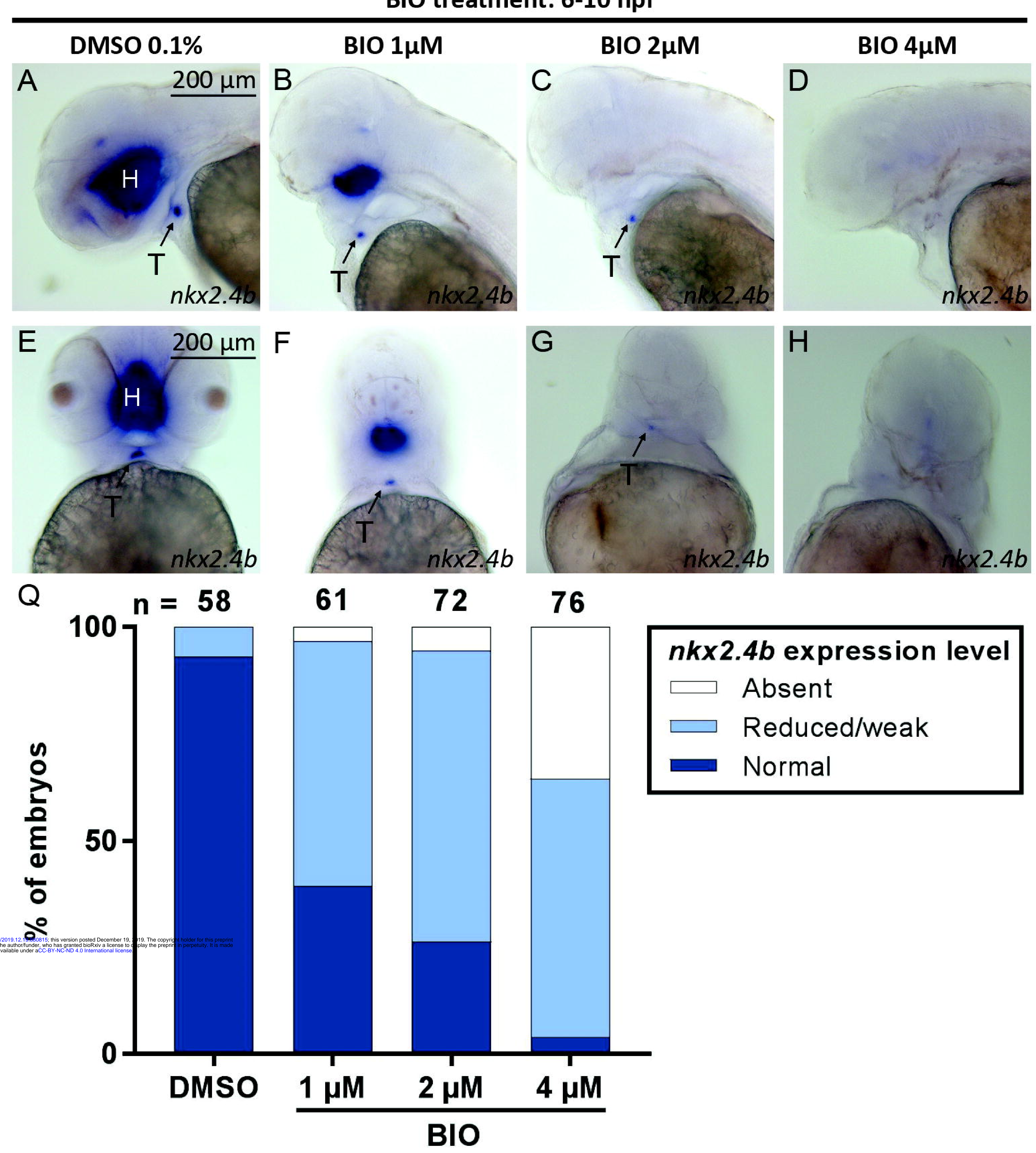

BIO treatment: 6-10 hpf
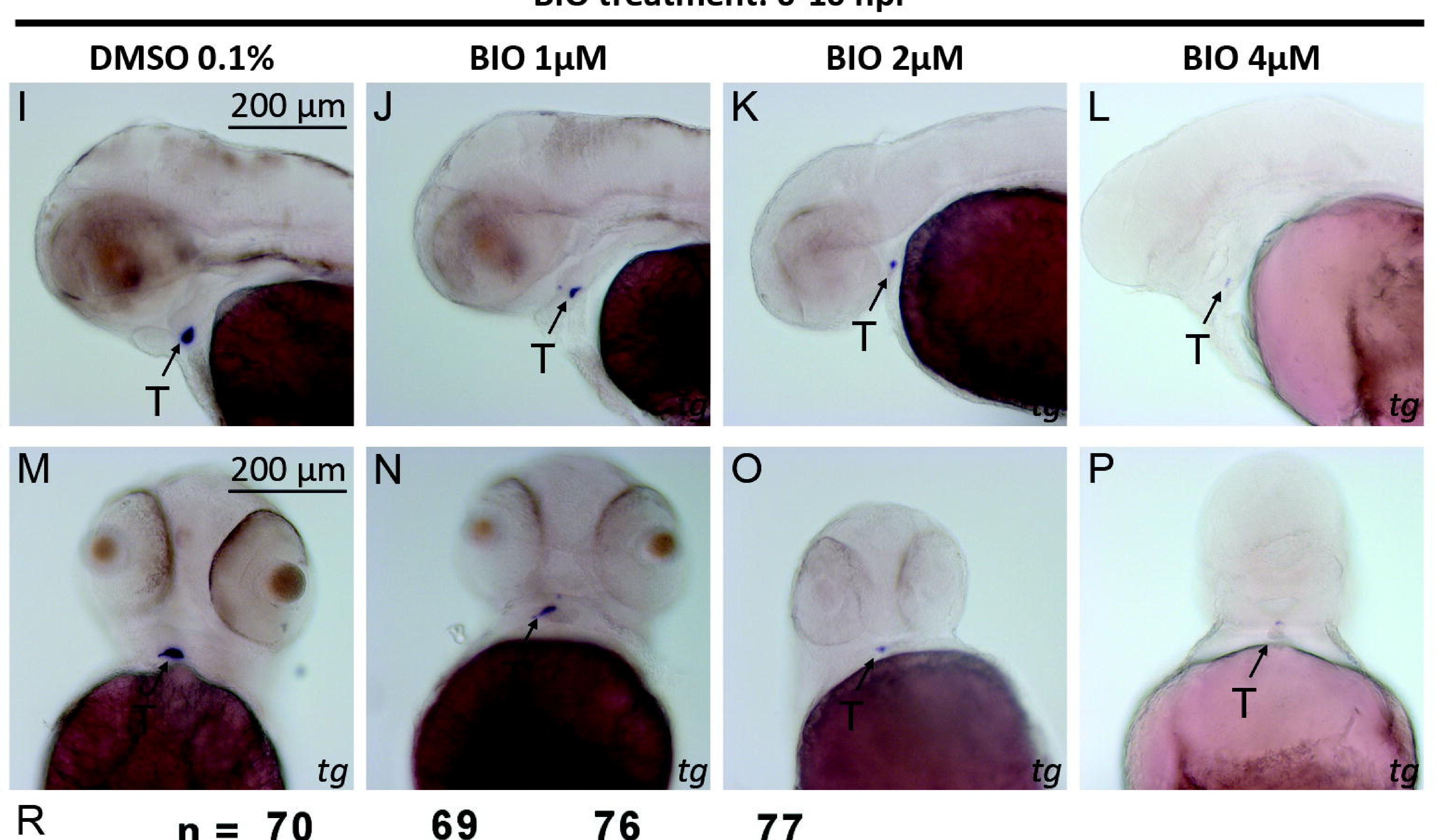

P

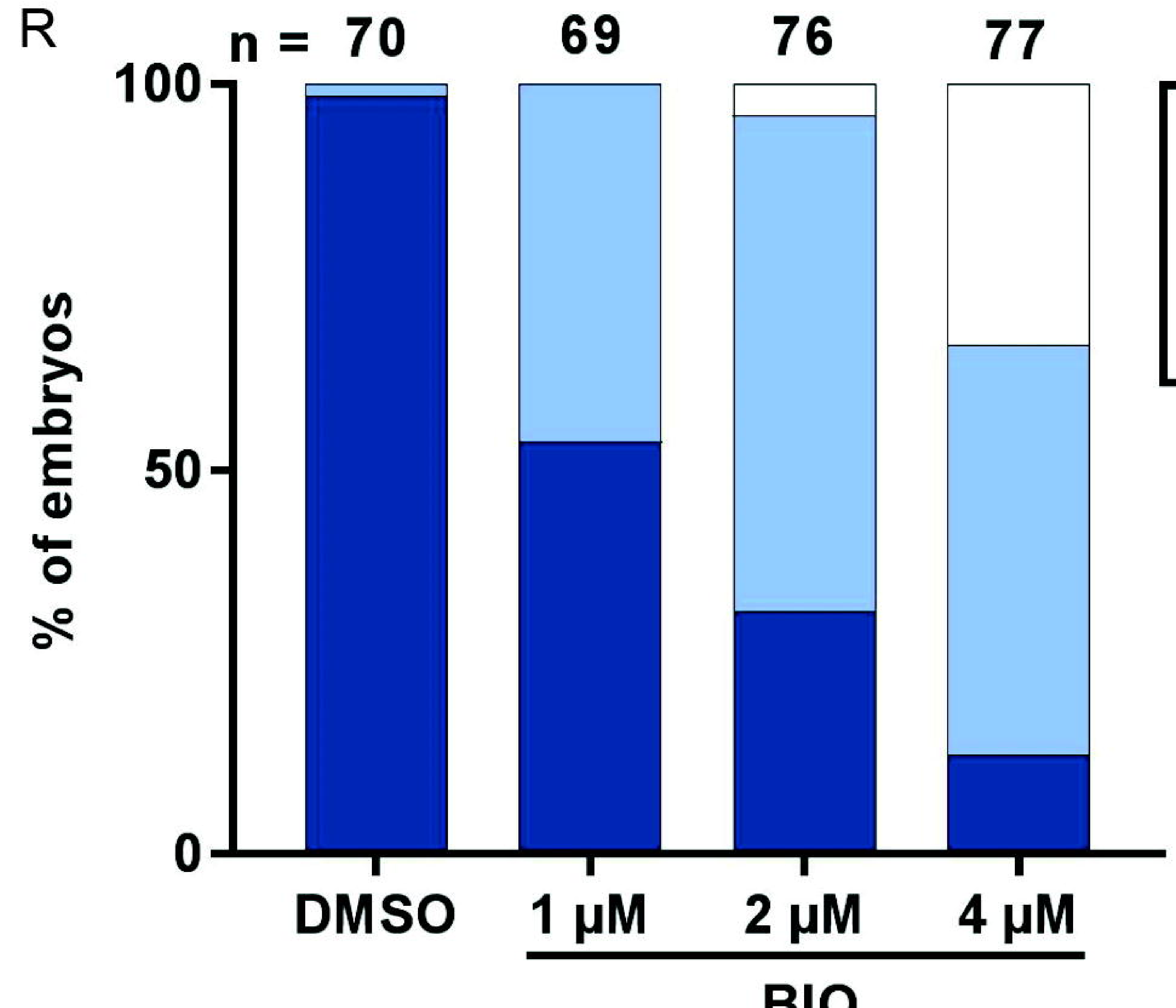

g expression level

$\square$ Absent

$\square$ Reduced/weak

- Normal 


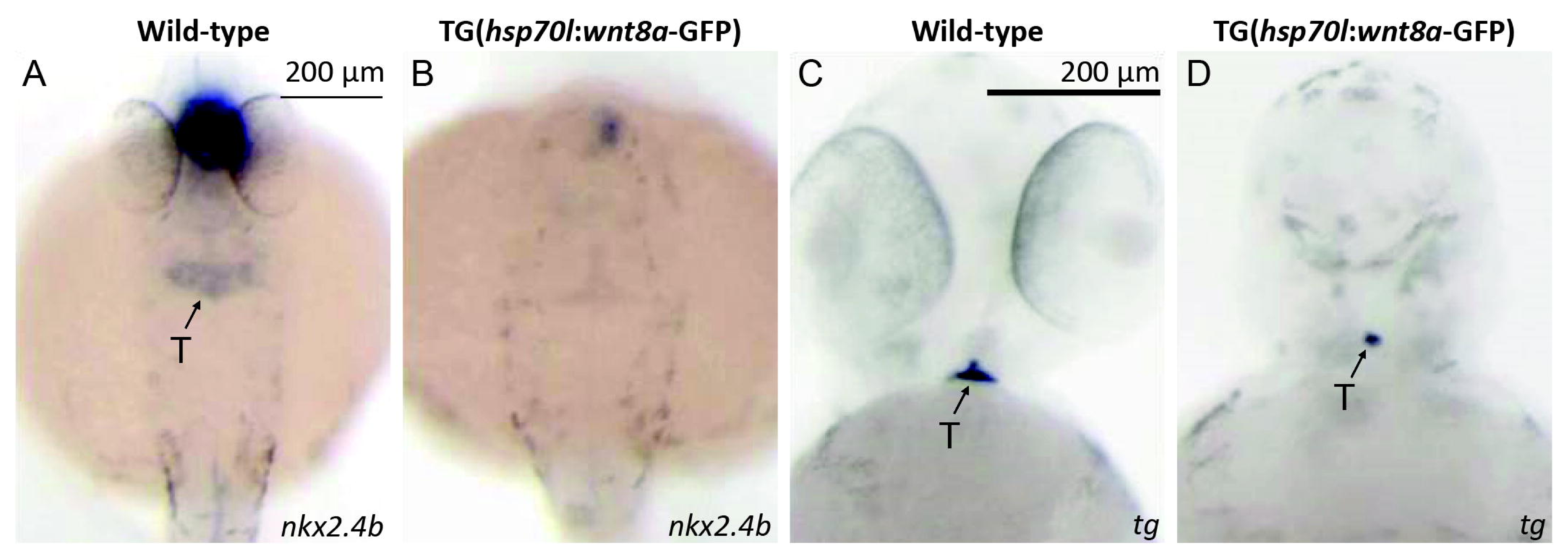




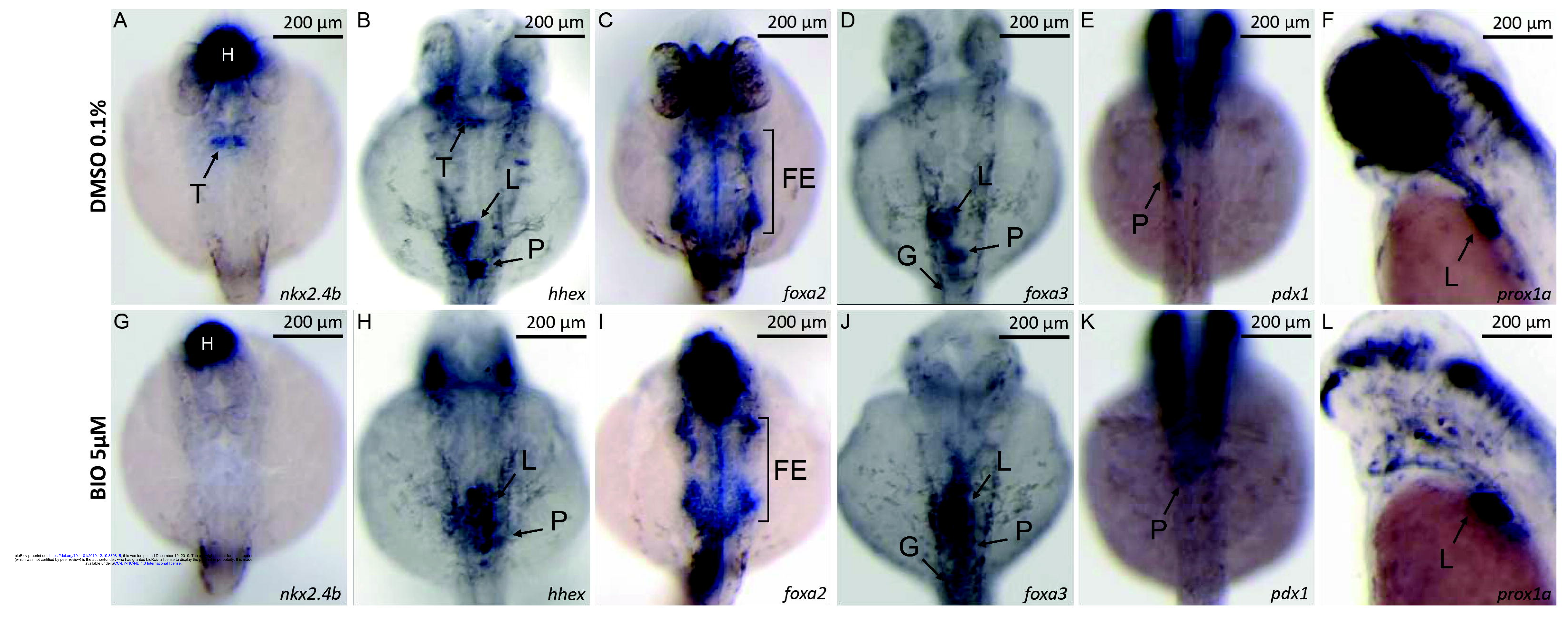



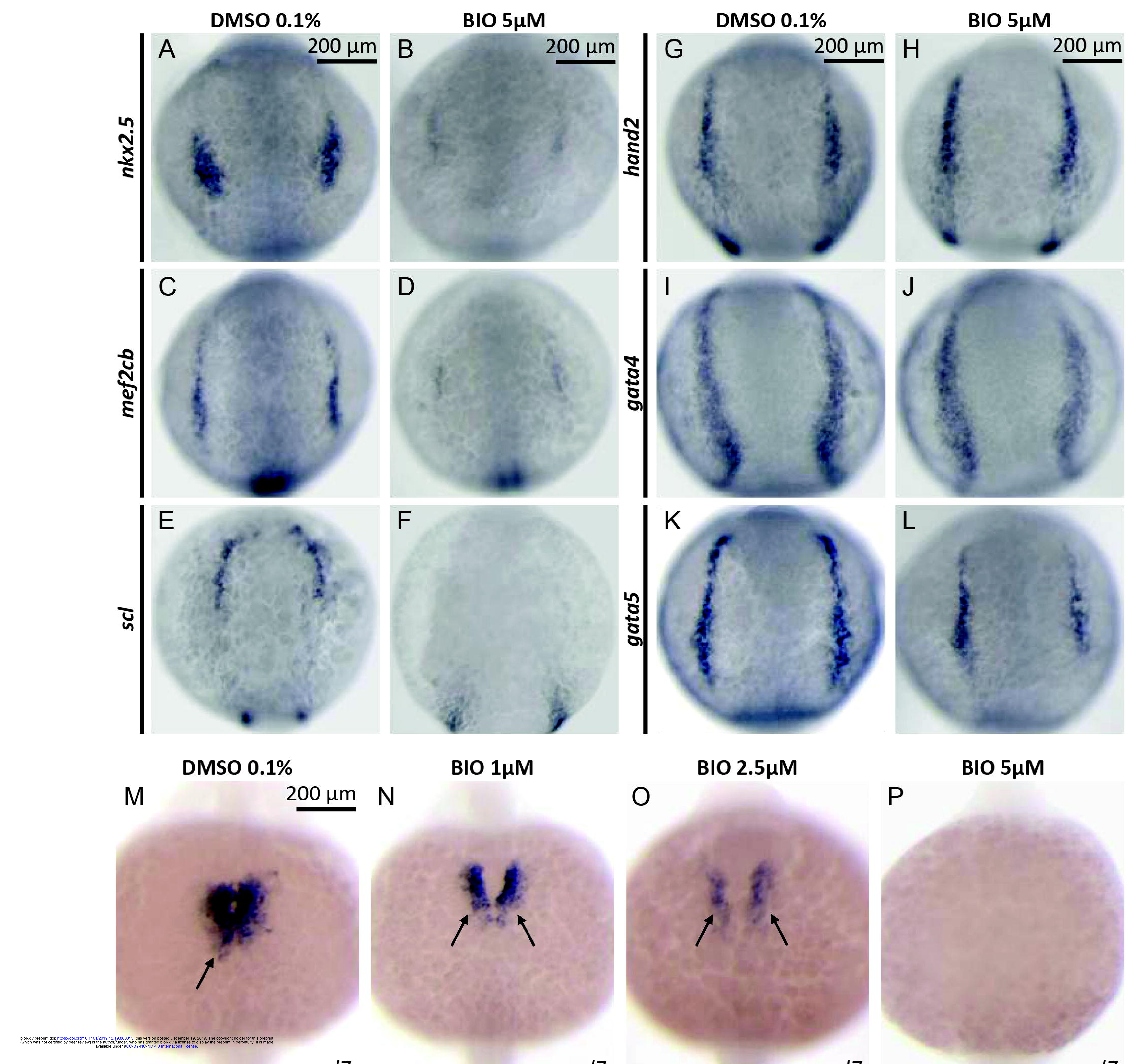

myl7
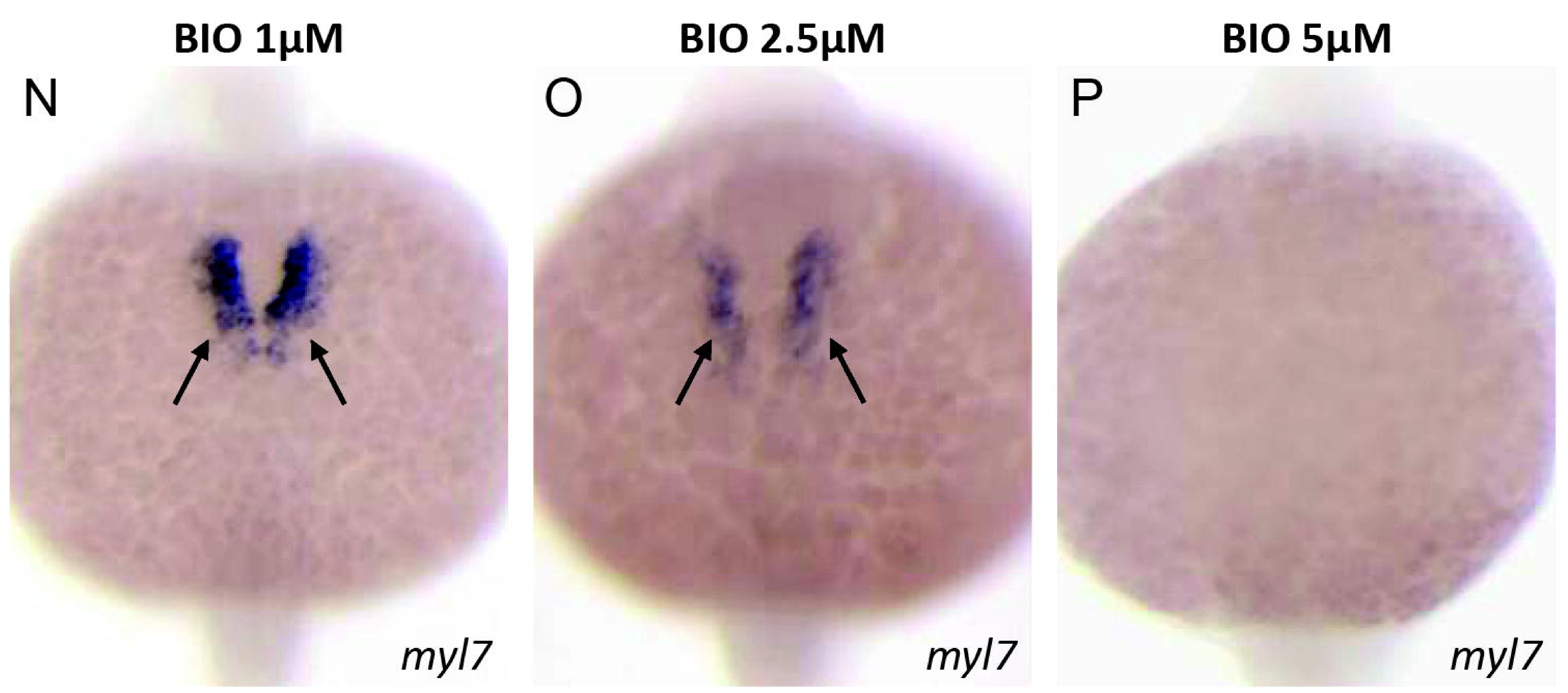
BIO treatment: $6-10 \mathrm{hpf}$



AZA treatment: 9-12 hpf

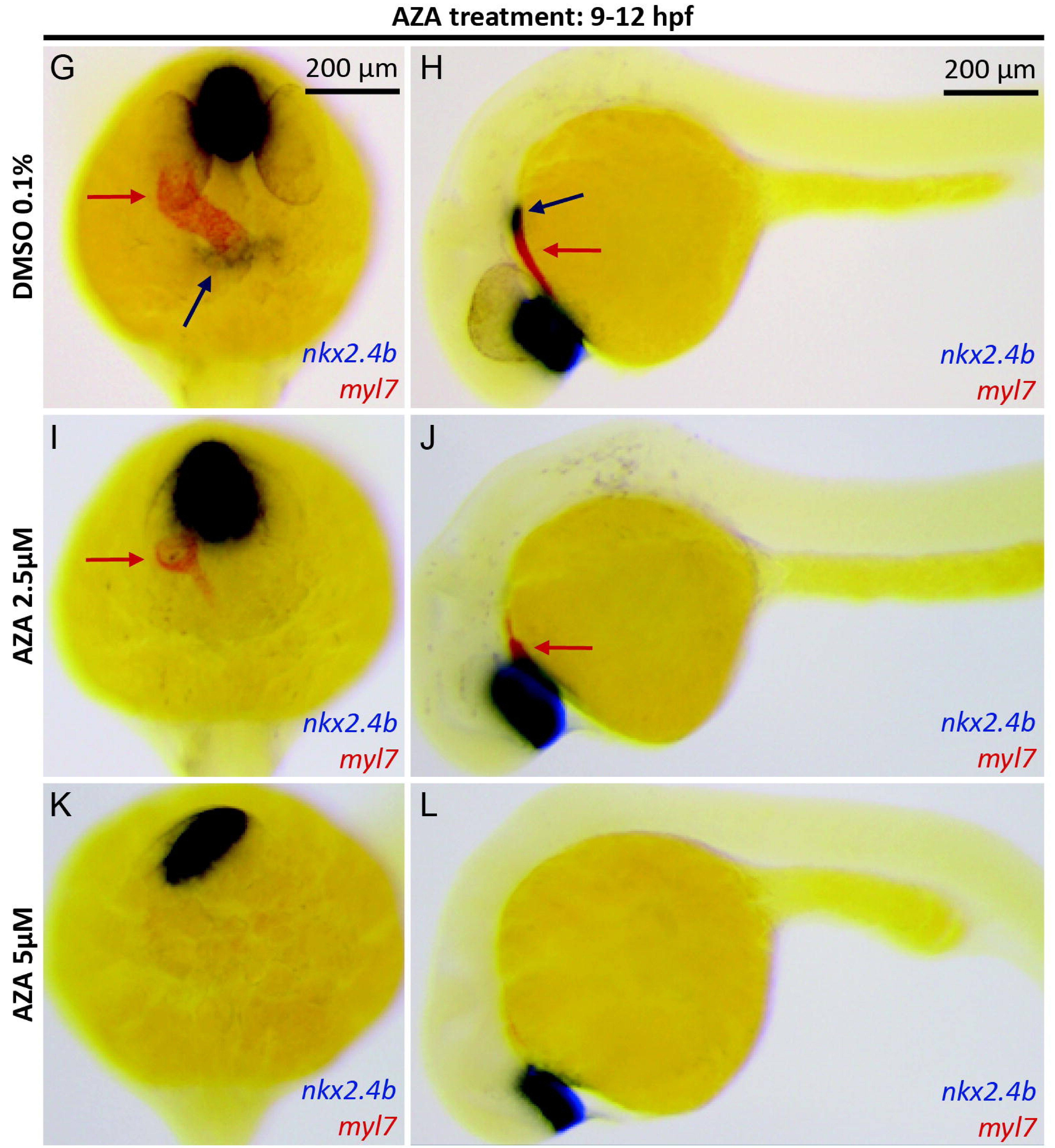



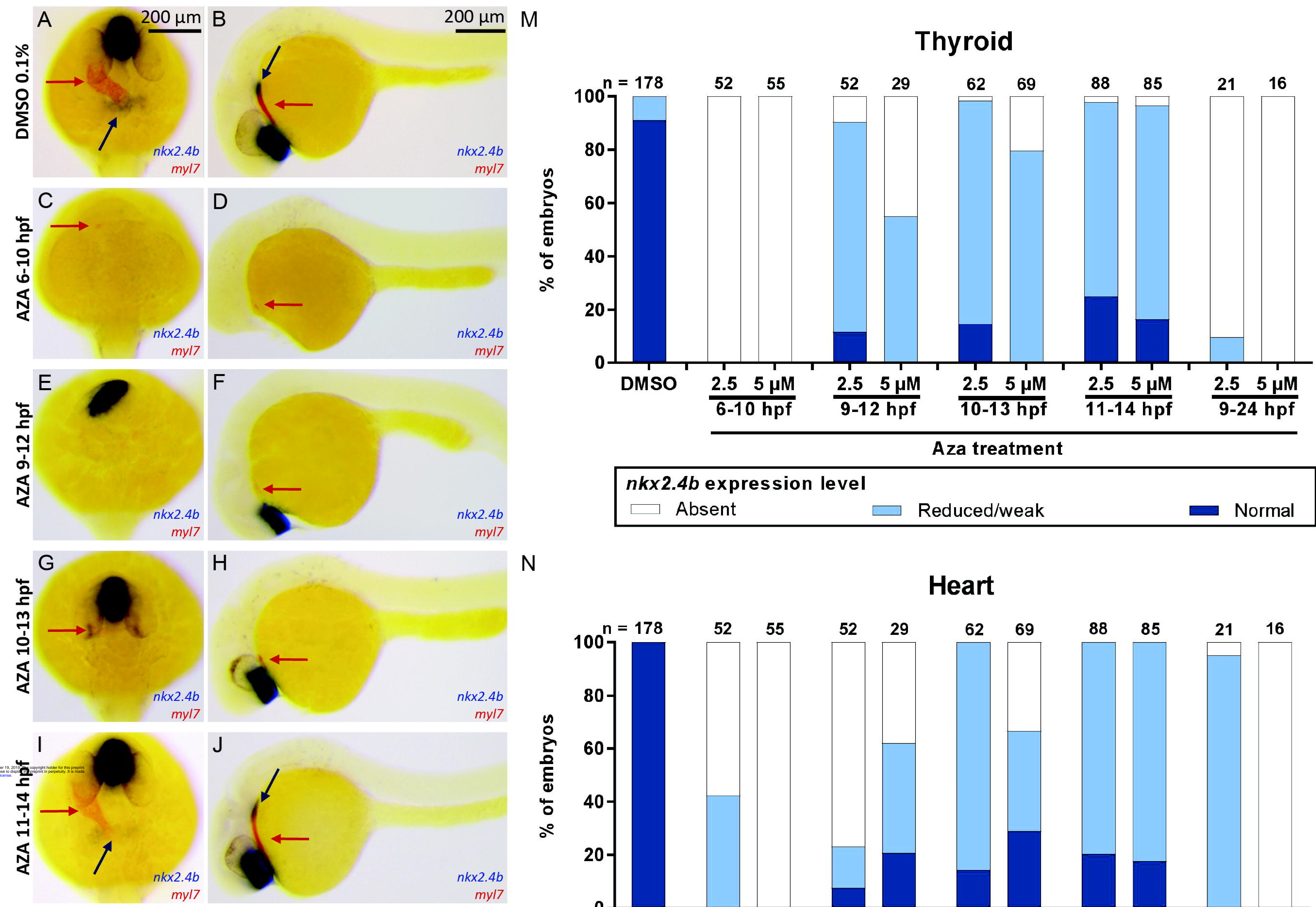

$\mathrm{N}$

Heart

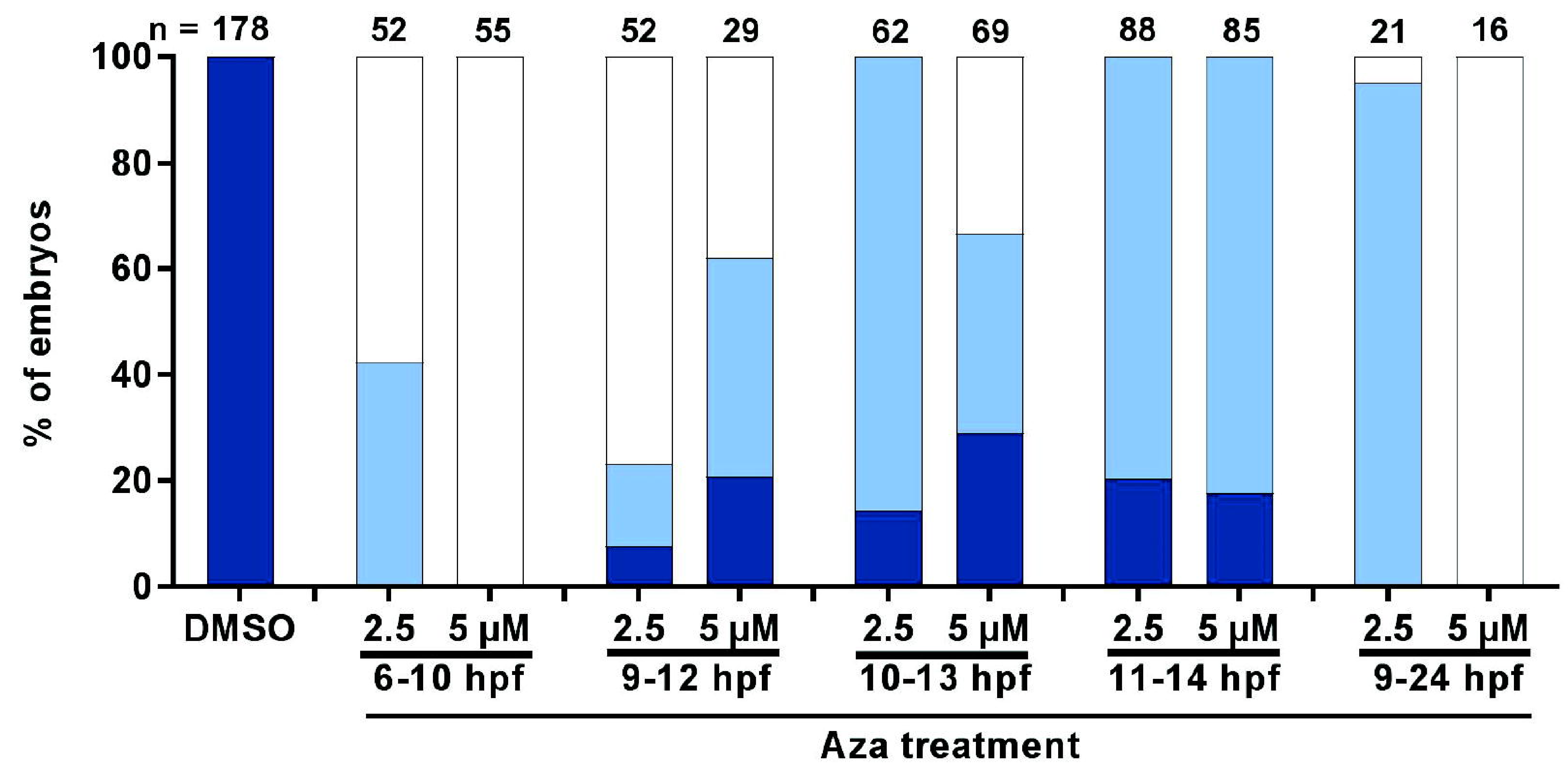

myl7 expression level

$\square$ Absent

$\square$ Reduced/weak

Normal 


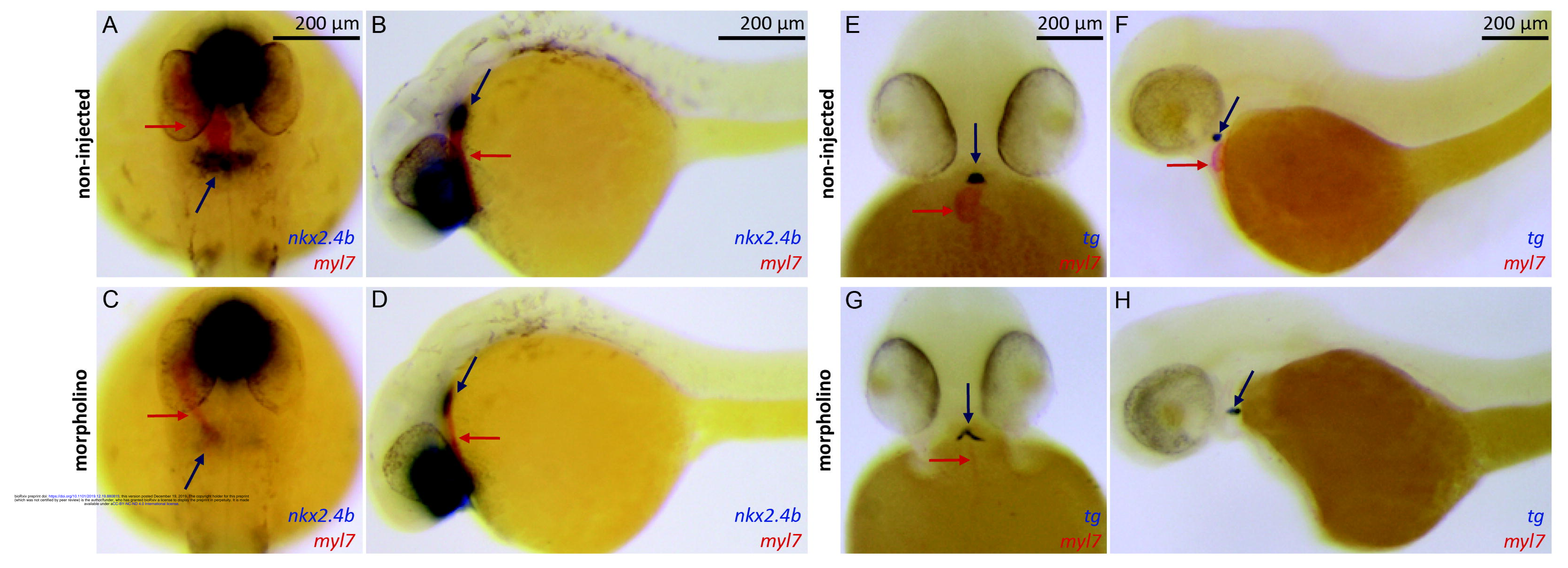



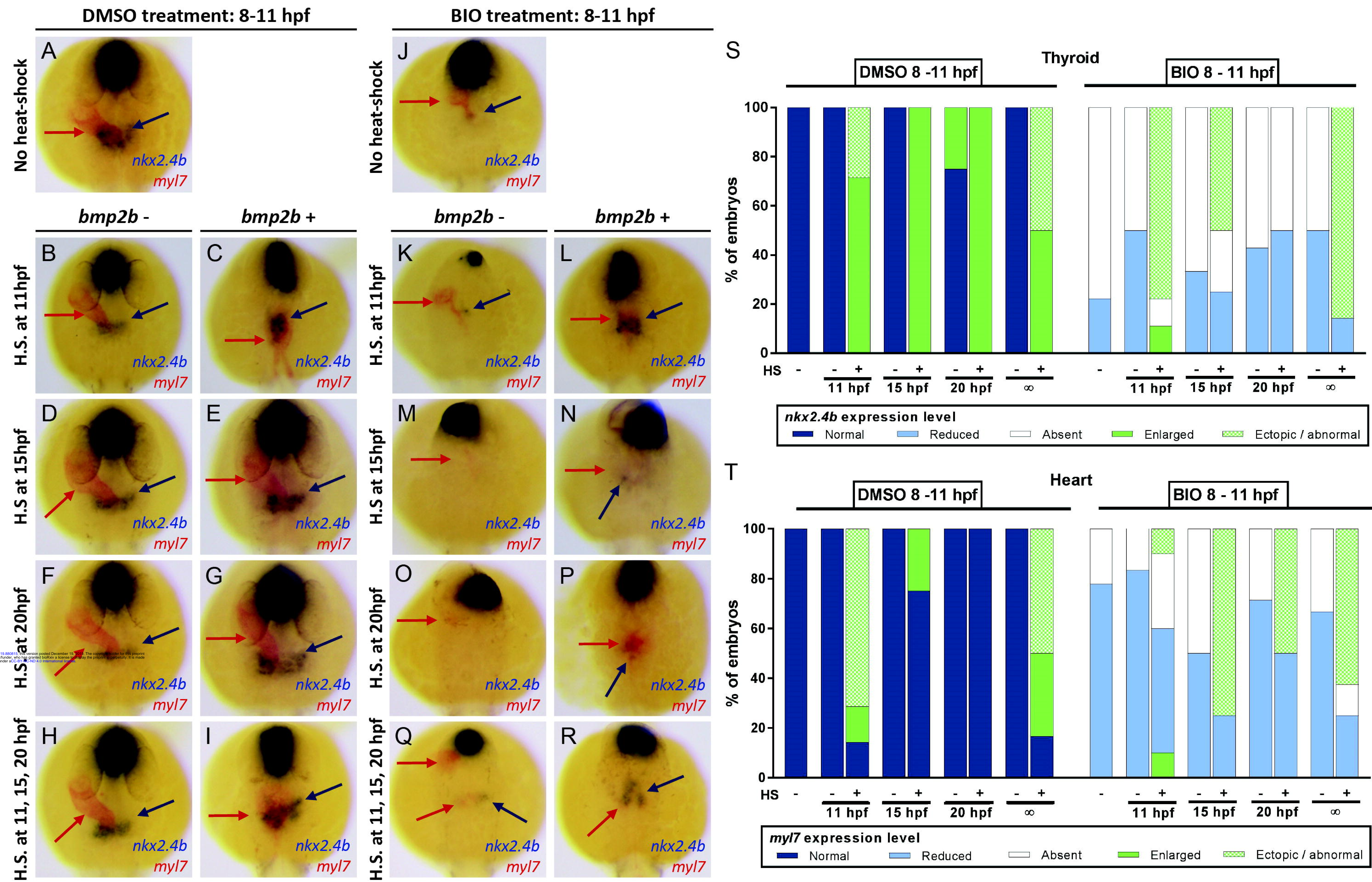

